\title{
Nonlinear wave growth theory of whistler-mode chorus and hiss emissions in the magnetosphere
}

\author{
Yoshiharu Omura* $\mathbb{0}$
}

\begin{abstract}
Nonlinear processes associated with the generation process of whistler-mode chorus emissions are summarized. The nonlinear dynamics of energetic electrons interacting with a coherent whistler-mode wave and the formation of electromagnetic electron holes or hills in the velocity phase space are described. The condition for resonant electrons to be free from the anomalous trapping at low pitch angles is obtained. In the presence of the inhomogeneity due to the frequency variation and the gradient of the magnetic field, the electron holes or hills result in resonant currents generating rising-tone emissions or falling-tone emissions, respectively. After formation of a coherent wave at a frequency of the maximum linear growth rate, triggering of the nonlinear wave growth takes place when the wave amplitude is above the threshold amplitude. The wave grows to a level close to the optimum wave amplitude as an absolute instability near the magnetic equator. The nonlinear growth rate at a position away from the equator is derived for a subtracted Maxwellian momentum distribution function with correction to the formulas in the past publications. The triggering process is repeated sequentially at progressively higher frequencies in the case of a risingtone emission, generating subpackets forming a chorus element. With a higher plasma density as in the plasmasphere, the triggering of subpackets takes place concurrently over a wide range of frequency forming discrete hiss elements with varying frequencies. The mechanism of nonlinear wave damping due to quasi-parallel propagation from the equator is presented, which results in the formation of a gap at half the electron cyclotron frequency, separating a long rising-tone chorus emission into the upper-band and lower-band chorus emissions. The theoretical formulation of an oblique whistler mode wave and its interaction with energetic electrons at the $n$-th resonance is also presented along with derivation of the inhomogeneity factor.
\end{abstract}

Keywords: Nonlinear, Wave-particle interaction, Instability, Whistler-mode wave, Acceleration, Coherent wave

\section{Introduction}

Whistler-mode chorus emissions have been studied for more than half a century, and their generation mechanism has not been clarified completely yet. However, there has been a substantial progress in our understanding of whistler-mode chorus emissions, thanks to successful reproduction of rising-tone and fallingtone emissions by particle simulations where nonlinear

*Correspondence: omura@rish.kyoto-u.ac.jp

Research Institute for Sustainable Humanosphere, Kyoto University, Uji, Kyoto 611-0011, Japan dynamics of resonant electrons are solved by combining Maxwell's equations and relativistic equations of motion for many energetic electrons (Katoh and Omura 2007, 2013; Katoh et al. 2018; Hikishima et al. 2009; Tao 2014; Ke et al. 2017; Lu et al. 2019; Nogi et al. 2020). While chorus emissions grow from thermal noise with the linear growth rate driven by high temperature anisotropy, triggered rising-tone emissions are excited by a coherent wave packet injected into the equatorial magnetosphere (Omura et al. 1991). The rising-tone emissions show nearly the same features of chorus emissions, and they have been reproduced by particle simulations (Hikishima 
et al. 2010; Hikishima and Omura 2012). A set of equations describing the frequency sweep rate and the nonlinear growth rate from the analysis of resonant currents formed by the nonlinear trapping potential of a coherent wave (Omura et al. 2008, 2009) have been derived. Nonlinear wave growth as an absolute instability becomes possible when the wave amplitude is higher than the threshold wave amplitude. The simple integration of the equations cannot reproduce some features of chorus emissions. Close examination of chorus emissions has revealed that a chorus rising-tone element with a wide frequency variation consists of many sub-packets with short and intense rising-tone emissions (Santolik et al. 2014). It is found that the resonant current parallel to the wave magnetic field also plays an important role in inducing variation of the frequency from that of the triggering wave (Omura and Nunn 2011). The wave growth is due to the frequency variation, which is controlled by the wave amplitude. The optimum wave amplitude has been derived based on the optimum condition for the nonlinear wave growth. Waves cannot grow much beyond the optimum wave amplitude.

Fine structure consisting of short rising and falling tones is also found in plasmaspheric hiss (Summers et al. 2014). There have been two different scenarios of the generation process. One is to assume external source of waves in the form of chorus emissions generated outside the plasmasphere (Bortnik et al. 2008). This mechanism was necessary to compensate for the small linear growth rate in the plasmasphere. The linear growth rates are too small to account for the large amplitude of the hiss emissions. It has not been explained, however, how the discrete chorus elements turn into the nearly continuous waves with very dense spectra filling the plasmasphere. As shown in Summers et al. (2014) and Nakamura et al. (2016, 2018), hiss emissions have fine structure with clear polarization, ellipticity, and wave normal angles close to those of the parallel propagation. The nonlinear wave growth theory for chorus emissions is applied to explanation of the fine structure, and a good agreement is found between the theory and the observation (Omura et al. 2015a) regarding the frequency ranges and wave amplitudes of hiss emissions.

Chorus emissions generated near the equator propagate to higher latitudes and their wave normal angles deviate to oblique directions. In the oblique propagation, there occurs the Landau resonance, and nonlinear trapping of energetic electrons takes place in addition to the cyclotron resonance trapping. Especially, trapping of energetic electrons due to the Landau resonance occurs effectively near half the cyclotron frequency, where the group velocity and the phase velocity become equal in the quasi-parallel propagation (Omura et al. 2009, 2019). Along with the gradient of the background magnetic field, the trapped electrons are accelerated by the parallel and perpendicular electric fields of the wave, while the waves near half the cyclotron frequency undergo damping giving energy to the electrons. This results in the formation of a gap separating chorus elements into the lower-band and upper-band emissions. Another factor to separate the lower-band and upper-band chorus is the propagation effect. There exists the Gendrin angle, at which the group velocity of oblique whistler-mode waves becomes parallel to the background magnetic field, for the lower band, while the Gendrin angle does not exist for the upper-band. Therefore, the propagation paths of the lower-band and upper-band chorus emissions are different.

This article is not intended to review all recent developments of studies on chorus and hiss emissions. Review papers on chorus emissions (Tao et al. 2020) and on controlled excitation of nonlinear wave-particle interactions (Golkowski et al. 2019) were published recently. Thanks to these comprehensive review papers, I can focus on providing a consistent summary of the nonlinear wave growth theory developed in recent years in an attempt to understand results of simulations and observations of whistlermode chorus and hiss emissions. Section 2 summarizes the dynamics of resonant electrons interacting with whistlermode waves propagating parallel to the parabolic magnetic field near the magnetic equator. The condition for resonant electrons to be free from the anomalous trapping at low pitch angles is also derived. Derivation of the nonlinear growth rate, threshold wave amplitude, and optimum wave amplitude for chorus and hiss emissions are presented in Sect. 3. Some inconsistencies of formulas found in the previous papers are corrected. The nonlinear growth rate at a position away from the equator is first obtained for a subtracted Maxwellian momentum distribution function. The dynamics of electrons interacting with an oblique whistler mode wave is described in Sect. 4. The inhomogeneity factor $S_{n}$ for the $n$-th resonance is also obtained for the first time as a function of the frequency sweep rate and the gradient of the background magnetic field. Section 5 gives a summary and discussion.

\section{Dynamics of resonant electron interacting with parallel whistler-mode waves \\ 2.1 Coherent waves}

We assume a whistler-mode wave propagating parallel to the static magnetic field $\boldsymbol{B}_{0}$. Its property as a wave is given by an amplitude $B_{\mathrm{w}}$ and the phase $\psi$. Based on the 
derivatives in space and time, we define the wave number and the frequency as

$$
k=-\frac{\partial \psi}{\partial h},
$$

and

$$
\omega=\frac{\partial \psi}{\partial t},
$$

where $h$ is a distance along the magnetic field line. We define the magnetic equator at $h=0$. The frequency and and the wave number satisfy the dispersion relation of whistler-mode wave propagating in the parallel direction, which is given for a cold plasma as (e.g., Stix (1992))

$$
c^{2} k^{2}=\omega^{2}+\frac{\omega \omega_{\mathrm{pe}}^{2}}{\Omega_{\mathrm{e}}-\omega} .
$$

Here, $c, \Omega_{\mathrm{e}}$, and $\omega_{\mathrm{pe}}$ are the speed of light, electron cyclotron frequency, and electron plasma frequency of the background cold plasma with a density $n_{\mathrm{c}}$, respectively. We simplify the dispersion relation by introducing the dimensionless parameters $\xi$ and $\chi(>0)$ satisfying the following relations:

$$
\xi^{2}=\frac{\omega\left(\Omega_{\mathrm{e}}-\omega\right)}{\omega_{\mathrm{pe}}^{2}},
$$

and

$$
\chi^{2}=1-\frac{\omega^{2}}{c^{2} k^{2}} .
$$

Then, the dispersion relation (3) is written as

$$
\chi^{2}=\frac{1}{1+\xi^{2}} .
$$

The phase velocity is conveniently expressed by a simple relation:

$$
V_{\mathrm{p}}=\frac{\omega}{k}=c \chi \xi .
$$

The group velocity is also given by

$$
V_{\mathrm{g}}=\frac{\partial \omega}{\partial k}=\frac{c \xi}{\chi}\left[\xi^{2}+\frac{\Omega_{\mathrm{e}}}{2\left(\Omega_{\mathrm{e}}-\omega\right)}\right]^{-1} .
$$

From the definitions of $k$ and $\omega$ given by (1) and (2), we have

$$
\frac{\partial k}{\partial t}=-\frac{\partial \omega}{\partial h} .
$$

Taking partial derivative of (3) in time and using (8) and (9), we obtain

$$
\frac{\partial \omega}{\partial t}+V_{\mathrm{g}} \frac{\partial \omega}{\partial h}=0 .
$$

This equation implies that the wave frequency is constant in the frame of reference moving with the group velocity. Therefore, the frequency of a wave packet does not change through propagation (Omura et al. 2008). As we will see later, the wave frequency only changes at the time of new wave packet formation through the absolute instability that takes place near the magnetic equator.

Figure 1 shows variation of the phase velocity $V_{\mathrm{p}}$ and the group velocity $V_{\mathrm{g}}$ as functions of frequency. At $\omega=0.5 \Omega_{\mathrm{e}}$, both velocities become equal. Namely, in the frame of reference moving with the group velocity, the wave phase becomes stationary. This property is very important for wave-particle interaction through Landau resonance, resulting in formation of upperband and lower-band chorus emissions as discussed in Sect. 4.

\subsection{Wave particle interaction with a coherent wave}

For simplicity, we assume only a single wave packet whose wave phase as observed by a particle is smoothly changing in space and time. We can call the wave as coherent, and under the coherent wave with a finite wave amplitude, the trajectories of resonant electrons undergo nonlinear motion as described by the relativistic equations of motion:

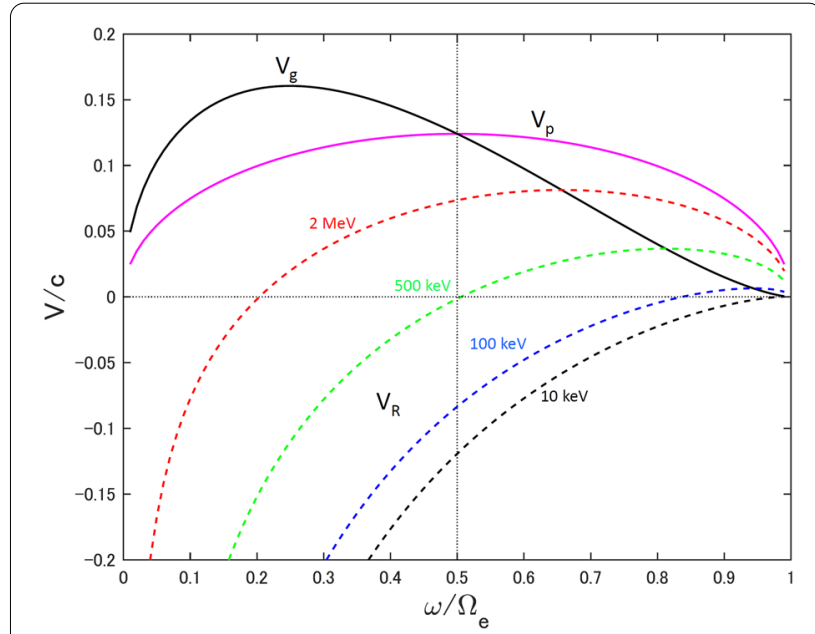

Fig. 1 Variation of $V_{g}, V_{p}$, and $V_{R}$. Group velocity $V_{g}$ in black solid line, phase velocity $V_{p}$ in magenta, and resonance velocities $V_{R}$ in dashed line for different energies $K=10 \mathrm{keV}$ (black), $100 \mathrm{keV}$ (blue), $500 \mathrm{keV}$ (green), and $2 \mathrm{MeV}$ (red) as functions of frequency $\omega$ with the plasma frequency $\omega_{\text {pe }}=4 \Omega_{\mathrm{e}}$ 


$$
m_{0} \frac{\mathrm{d}(\gamma \boldsymbol{v})}{\mathrm{d} t}=-e\left[\boldsymbol{E}_{\mathrm{w}}+\boldsymbol{v} \times\left(\boldsymbol{B}_{0}+\boldsymbol{B}_{\mathrm{w}}\right)\right],
$$

where $m_{0}$ is the electron rest mass, and $\gamma$ is the Lorentz factor given by

$$
\gamma=\left[1-\frac{v_{\|}^{2}+v_{\perp}^{2}}{c^{2}}\right]^{-1 / 2} .
$$

We have assumed an electron moving along the $h$-axis parallel to the background magnetic field $\boldsymbol{B}_{0}$ with a parallel velocity $v_{\|}$and a perpendicular velocity $\vec{v}_{\perp}=v_{\perp} \exp (i \phi)$, where a complex number is used to represent the phase of the perpendicular velocity vector $\boldsymbol{v}_{\perp}$ in the transverse plane. The background magnetic field satisfies $\nabla \cdot \boldsymbol{B}_{0}=0$, which is written in cylindrical coordinates with the axis at the center of the cyclotron motion of an electron:

$$
\frac{1}{r} \frac{\partial\left(r B_{\mathrm{r}}\right)}{\partial r}+\frac{\partial B_{0}}{\partial h}=0,
$$

where $r$ is a radial distance from the center of the cyclotron motion, and the cyclotron radius is given by $r_{\mathrm{c}}=\gamma v_{\perp} / \Omega_{\mathrm{e}}$. Integrating (13) from 0 to $r_{\mathrm{c}}$, we obtain a radial component of the background magnetic field at the position of the particle:

$$
B_{\mathrm{r}}=-\frac{r_{\mathrm{c}}}{2} \frac{\partial B_{0}}{\partial h} .
$$

We assume the wavenumber vector $\boldsymbol{k}$ is in the same direction as the background magnetic field $\boldsymbol{B}_{0}$, and the wave becomes a purely transverse wave with $E_{\mathrm{w} \|}=0$ and $B_{\mathrm{w} \|}=0$. We decompose $\boldsymbol{v}, \boldsymbol{B}_{\mathrm{w}}$, and $\boldsymbol{E}_{\mathrm{w}}$, into components parallel and perpendicular to $\boldsymbol{B}_{0}$ as $\boldsymbol{v}=\left[\nu_{\|}, v_{\perp} \mathrm{e}^{i \phi}\right]$, $\boldsymbol{B}_{\mathrm{w}}=\left[0, B_{\mathrm{w}} \mathrm{e}^{i \psi}\right]$, and $\boldsymbol{E}_{\mathrm{w}}=\left[0, E_{\mathrm{w}} \mathrm{e}^{i(\psi-\pi / 2)}\right]$, respectively. We have expressed the vectors perpendicular to $\boldsymbol{B}_{0}$ by complex numbers. Inserting these elements of vectors into (11), we have

$$
\begin{aligned}
m_{0} \frac{\mathrm{d}\left(\gamma v_{\|}\right)}{\mathrm{d} t}=-e\left(-v_{\perp} B_{\mathrm{w}} \sin \zeta-v_{\perp} B_{\mathrm{r}}\right) \\
\begin{aligned}
m_{0} \frac{\mathrm{d}\left(\gamma v_{\perp} \mathrm{e}^{i \phi}\right)}{\mathrm{d} t}= & -e\left[\left(v_{\|} B_{\mathrm{w}}-E_{\mathrm{w}}\right)(\sin \zeta+i \cos \zeta)\right. \\
& \left.+v_{\|} B_{\mathrm{r}}-i v_{\perp} B_{0}\right] \mathrm{e}^{i \phi}
\end{aligned}
\end{aligned}
$$

where $\zeta=\phi-\psi$.

Noting that the amplitude $E_{\mathrm{w}}$ of the wave electric field is expressed by the amplitude $B_{\mathrm{w}}$ of the wave magnetic field as $E_{\mathrm{w}}=(\omega / k) B_{\mathrm{w}}$, the equation of motion (15) is rewritten as

$$
\frac{\mathrm{d}\left(\gamma v_{\|}\right)}{\mathrm{d} t}=v_{\perp} \Omega_{\mathrm{w}} \sin \zeta-\frac{\gamma v_{\perp}^{2}}{2 \Omega_{\mathrm{e}}} \frac{\partial \Omega_{\mathrm{e}}}{\partial h},
$$

where we normalize the wave magnetic field as $\Omega_{\mathrm{w}}=e B_{\mathrm{w} \perp} / m_{0}$. Separating the real and imaginary parts of (16) divided by $\mathrm{e}^{i \phi}$, we obtain

$$
\frac{\mathrm{d}\left(\gamma v_{\perp}\right)}{\mathrm{d} t}=\left(\frac{\omega}{k}-v_{\|}\right) \Omega_{\mathrm{w}} \sin \zeta+\frac{\gamma v_{\|} v_{\perp}}{2 \Omega_{\mathrm{e}}} \frac{\partial \Omega_{\mathrm{e}}}{\partial h},
$$

and

$$
\frac{\mathrm{d} \phi}{\mathrm{d} t}=\frac{1}{\gamma v_{\perp}}\left(\frac{\omega}{k}-v_{\|}\right) \Omega_{\mathrm{w}} \cos \zeta+\frac{\Omega_{\mathrm{e}}}{\gamma} .
$$

\subsection{Resonance conditions}

We take a time derivative of the relative phase angle $\zeta=\phi-\psi$ between the perpendicular velocity $\boldsymbol{v}_{\perp}$ of a particle and the wave magnetic field $\mathbf{B}_{\mathrm{w}}$ as observed from a frame of reference moving with the parallel velocity $v_{\|}$ of the particle. From (1) and (2), we have

$$
\frac{\mathrm{d} \zeta}{\mathrm{d} t}=\frac{\mathrm{d} \phi}{\mathrm{d} t}-\omega+k v_{\|} .
$$

The resonance condition between the wave and the electron is given by

$$
\omega-k V_{\mathrm{R}}=\frac{\Omega_{\mathrm{e}}}{\gamma} .
$$

Solving for $V_{R}$, we have

$$
V_{\mathrm{R}}=\left(1-\frac{\Omega_{\mathrm{e}}}{\gamma \omega}\right) V_{\mathrm{p}}
$$

When $v_{\|}=V_{\mathrm{R}}$, the electron is at the cyclotron resonance with the wave, and it undergoes strong deviation from the adiabatic orbit, giving energy to the wave, or receiving energy from the wave. We have plotted the variation of resonance velocities for different energies in Fig. 1. The resonance velocity is also calculated by specifying the resonance energy $K=(\gamma-1) 511 \mathrm{keV}$, which is also a function of $V_{\mathrm{R}}$. For a specific value of the perpendicular velocity $v_{\perp}$, we can express $V_{\mathrm{R}}$ as an explicit function from (7), (12) and (21) as

$$
\tilde{V}_{\mathrm{R}}=\frac{\tilde{\omega}^{2}-\sqrt{\tilde{\omega}^{4}+\left(\tilde{\omega}^{2}+\tilde{V}_{\mathrm{p}}^{2}\right)\left(1-\tilde{\omega}^{2}-\tilde{v}_{\perp}^{2}\right)}}{\tilde{\omega}^{2}+\tilde{V}_{\mathrm{p}}^{2}} \tilde{V}_{\mathrm{p}},
$$

where $\quad \tilde{\omega}=\omega / \Omega_{\mathrm{e}}, \quad \tilde{V}_{\mathrm{R}}=V_{\mathrm{R}} / c, \quad \tilde{v}_{\perp}=v_{\perp} / c, \quad$ and $\tilde{V}_{\mathrm{p}}=V_{\mathrm{p}} / c=\chi \xi$.

Substituting (19) and (21) into (20), we obtain 


$$
\frac{\mathrm{d} \zeta}{\mathrm{d} t}=\frac{1}{\gamma k v_{\perp}}\left(\frac{\Omega_{\mathrm{e}}}{\gamma}-\theta\right) \Omega_{\mathrm{w}} \cos \zeta+\theta,
$$

where $\theta=k\left(v_{\|}-V_{\mathrm{R}}\right)$. For electrons near the cyclotron resonance, $\theta$ varies with the order of the trapping frequency $\omega_{\mathrm{tr}}$, which is derived later as $\omega_{\mathrm{tr}}=\chi \sqrt{k v_{\perp} \Omega_{\mathrm{w}} / \gamma}$. Since $\chi \sim 1$ and $\theta \sim \omega_{\text {tr }}$, we rewrite the equation as

$$
\frac{\mathrm{d} \zeta}{\mathrm{d} t} \simeq \omega_{\mathrm{tr}}\left[\left(\frac{\Omega_{\mathrm{w}}}{\Omega_{\mathrm{e}}}\right)^{1 / 2}\left(\frac{\gamma k v_{\perp}}{\Omega_{\mathrm{e}}}\right)^{-3 / 2} \cos \zeta+1\right] .
$$

Using (22), we obtain the following condition

$$
\left(\frac{\Omega_{\mathrm{w}}}{\Omega_{\mathrm{e}}}\right)^{1 / 2} \ll\left(\frac{v_{\perp}}{V_{\mathrm{p}}-V_{\mathrm{R}}}\right)^{3 / 2}
$$

which ensures that we can neglect the first term on the right-hand side of (24). The resonance velocity $V_{\mathrm{R}}$ takes a negative value for electrons with energy less than 100 $\mathrm{keV}$, and it changes its sign for higher energies, approaching to $V_{\mathrm{p}}$ in the relativistic energy range, as shown in Fig. 1. The condition (26) is well satisfied in the case of chorus wave generation due to energetic electrons of $10 \sim 100 \mathrm{keV}$ with temperature anisotropy $\left(T_{\perp}>T_{\|}\right)$ and in the case of electron acceleration to MeV energy in the magnetosphere, because $V_{\mathrm{R}}$ approaches $V_{\mathrm{p}}$ with $\mathrm{MeV}$ energy. We thus obtain

$$
\frac{\mathrm{d} \zeta}{\mathrm{d} t}=\theta
$$

When $\theta \simeq 0$, i.e., $v_{\|} \simeq V_{\mathrm{R}}$, the first-order phase variation becomes very small, and this is the first-order cyclotron resonance condition.

When electrons with small pitch angles such as those precipitating into the loss cone interact with a very large amplitude wave, (26) is not satisfied, resulting in anomalous trapping of the electrons transporting them from the loss cone to larger pitch angles (Kitahara and Katoh 2019). When relativistic electrons at small pitch angles interact with electromagnetic ion cyclotron (EMIC) waves, effective scattering to lower pitch angles and into the loss cone takes place because of the same mechanism (Kubota and Omura 2017). The large pitch angle scattering is due to variation of the perpendicular velocity $v_{\perp}$ as indicated by (18). Taking into account the variation of $v_{\perp}$, Yoon and Bellan (2020) have made an elaborate analysis on dynamics of electrons interacting with a large amplitude wave with a constat frequency in a uniform magnetic field. They found significant scattering takes place for particles at low pitch angles near a specific gyrophase, which is due to the first terms on the right-hand sides of (18) and (19). The scattering at low pitch angles, however, does not contribute much to the formation of the resonant currents exciting chorus emissions. In the following analysis, we assume the perpendicular velocity $v_{\perp}$ is constant for simplicity. It should be noted that variation of the perpendicular velocity is taken into account in the test particle simulations (Hiraga and Omura 2020; Hsieh and Omura 2017,2018 ) and all self-consistent simulaitons of chorus and hiss emissions.

Assuming $\theta=v_{\|}-V_{\mathrm{R}} \simeq 0$, we take the second-order derivative of the relative phase angle $\zeta$ as

$$
\frac{\mathrm{d}^{2} \zeta}{\mathrm{d} t^{2}}=k\left(\frac{\mathrm{d} v_{\|}}{\mathrm{d} t}-\frac{\mathrm{d} V_{\mathrm{R}}}{\mathrm{d} t}\right)
$$

The first term on the right hand side of (28) is expanded using the equation of motion (17) as

$$
\frac{\mathrm{d} v_{\|}}{\mathrm{d} t}=\frac{\Omega_{\mathrm{w}} v_{\perp}}{\gamma} \sin \zeta-\frac{v_{\|}}{\gamma} \frac{\mathrm{d} \gamma}{\mathrm{d} t}-\frac{\nu_{\perp}^{2}}{2 \Omega_{\mathrm{e}}} \frac{\partial \Omega_{\mathrm{e}}}{\partial h} .
$$

The second term of (28) is the time variation of the resonance velocity as observed by a particle moving with a parallel velocity $v_{\|}$:

$$
\frac{\mathrm{d} V_{\mathrm{R}}}{\mathrm{d} t}=\frac{1}{k}\left(\frac{\mathrm{d} \omega}{\mathrm{d} t}-\frac{\nu_{\|}}{\gamma} \frac{\partial \Omega_{\mathrm{e}}}{\partial h}+\frac{\Omega_{\mathrm{e}}}{\gamma^{2}} \frac{\mathrm{d} \gamma}{\mathrm{d} t}\right)-\frac{1}{k^{2}}\left(\omega-\frac{\Omega_{\mathrm{e}}}{\gamma}\right) \frac{\mathrm{d} k}{\mathrm{~d} t} .
$$

Using the phase relation $\partial k / \partial t=-\partial \omega / \partial h$ and the wave equation (10), we can expand the time derivative of the wave number $k$ in the last term of (30) as

$$
\frac{\mathrm{d} k}{\mathrm{~d} t}=\frac{1}{V_{\mathrm{g}}} \frac{\partial \omega}{\partial t}+v_{\|} \frac{\partial k}{\partial h} .
$$

We differentiate the dispersion relation (3) by $h$ to obtain

$$
\frac{\partial k}{\partial h}=\frac{1}{V_{\mathrm{g}}} \frac{\partial \omega}{\partial h}-\Lambda \frac{\chi \omega}{2 c \xi\left(\Omega_{\mathrm{e}}-\omega\right)} \frac{\partial \Omega_{\mathrm{e}}}{\partial h} .
$$

We have defined the parameter $\Lambda$ to incorporate the inhomogeneous cold electron density model $N_{\mathrm{e}}(h)$ along the background magnetic field line as $N_{\mathrm{e}}(h)=N_{\mathrm{e} 0} \Omega_{\mathrm{e}}(h) / \Omega_{\mathrm{e} 0}$, where $N_{\mathrm{e} 0}$ and $\Omega_{\mathrm{e} 0}$ are the cold electron density and the electron cyclotron frequency at the equator, respectively. We have $\Lambda=\omega / \Omega_{\mathrm{e}}$ for the inhomogeneous model (Omura et al. 2009), while $\Lambda=1$ for the constant cold electron density model (Omura et al. 2008). From (10), (30), (31), and (32), we obtain

$$
\begin{aligned}
\frac{\mathrm{d} V_{\mathrm{R}}}{\mathrm{d} t}= & \frac{\Omega_{\mathrm{e}}}{k \gamma^{2}} \frac{\mathrm{d} \gamma}{\mathrm{d} t}+\frac{1}{k}\left(1-\frac{V_{\mathrm{R}}}{V_{\mathrm{g}}}\right)\left(1-\frac{\nu_{\|}}{V_{\mathrm{g}}}\right) \frac{\partial \omega}{\partial t} \\
& -\frac{\nu_{\|}}{\gamma k}\left[1+\Lambda \frac{\chi^{2}\left(\Omega_{\mathrm{e}}-\gamma \omega\right)}{2\left(\Omega_{\mathrm{e}}-\omega\right)}\right] \frac{\partial \Omega_{\mathrm{e}}}{\partial h},
\end{aligned}
$$


where we have used the wave equation (10). The variation of the Lorentz factor can be estimated from the variation of the kinetic energy $K$ of an electron

$$
\frac{\mathrm{d} K}{\mathrm{~d} t}=-e v_{\perp} E_{\mathrm{w}} \cos \left(\zeta+\frac{\pi}{2}\right)=e v_{\perp} E_{\mathrm{w}} \sin \zeta .
$$

From the formula of the relativistic kinetic energy $K=m_{0} c^{2}(\gamma-1)$ and Maxwell's equation $E_{\mathrm{w}}=(\omega / k) B_{\mathrm{w}}$, we obtain

$$
\frac{\mathrm{d} \gamma}{\mathrm{d} t}=\frac{\omega \Omega_{\mathrm{w}} \nu_{\perp}}{k c^{2}} \sin \zeta
$$

where $\Omega_{\mathrm{w}}$ is a wave amplitude normalized by the electron cyclotron frequency, and it is given by $\Omega_{\mathrm{w}}=e B_{\mathrm{w}} / m_{0}$. Substituting (29), (33), and (35) into (28), we obtain the second-order nonlinear ordinary differential equaiton of the phase angle as seen from the electron:

$$
\frac{\mathrm{d}^{2} \zeta}{\mathrm{d} t^{2}}=\frac{\omega_{t}^{2} \chi^{2}}{\gamma}(\sin \zeta+S) .
$$

The secular term $S$ is the inhomogeneity factor given by

$$
S=-\frac{1}{s_{0} \omega \Omega_{\mathrm{w}}}\left(s_{1} \frac{\partial \omega}{\partial t}+c s_{2} \frac{\partial \Omega_{\mathrm{e}}}{\partial h}\right),
$$

where

$$
\begin{aligned}
& s_{0}=\frac{\chi v_{\perp}}{\xi c}, \\
& s_{1}=\gamma\left(1-\frac{V_{\mathrm{R}}}{V_{\mathrm{g}}}\right)^{2},
\end{aligned}
$$

and

$$
s_{2}=\frac{1}{2 \xi \chi}\left\{\frac{\gamma \omega}{\Omega_{\mathrm{e}}}\left(\frac{\nu_{\perp}}{c}\right)^{2}-\left[2+\Lambda \frac{\chi^{2}\left(\Omega_{\mathrm{e}}-\gamma \omega\right)}{\Omega_{\mathrm{e}}-\omega}\right] \frac{V_{\mathrm{R}} V_{\mathrm{p}}}{c^{2}}\right\} .
$$

Here we have defined the trapping frequency $\omega_{t}=\sqrt{k v_{\perp} \Omega_{\mathrm{w}}}$. In the non-relativistic limit of $\gamma=1$ and $\chi=1\left(V_{\mathrm{p}} \ll c\right)$, electrons trapped in the wave potential oscillate with $\omega_{t}$ near the stable equilibrium point of $(\zeta, \theta)=(\pi, 0)$ in a homogeneous medium with $S=0$.

We rewrite (27) and (36) as a set of equations describing nonlinear motion of a resonant electron under a coherent wave as

$$
\begin{aligned}
& \frac{\mathrm{d} \zeta}{\mathrm{d} t}=\theta, \\
& \frac{\mathrm{d} \theta}{\mathrm{d} t}=\omega_{\mathrm{tr}}^{2}(\sin \zeta+S),
\end{aligned}
$$

where $\omega_{\mathrm{tr}}=\omega_{t} \chi / \sqrt{\gamma}$. When the right-hand side of (41) is zero, i.e., $\mathrm{d} \zeta / \mathrm{d} t=0$, the first-order resonance condition is satisfied. In addition, when the right-hand side of (42) is zero, i.e., $\mathrm{d} \theta / \mathrm{d} t=\mathrm{d}^{2} \zeta / \mathrm{d} t^{2}$, the second-order resonance condition is satisfied. When $|S| \leq 1$, the secondorder resonance condition is satisfied at two phase angles $\zeta_{0}$ closer to $\pi$ and $\zeta_{1}$ closer to 0 . The point $\left(\zeta_{0}, 0\right)$ in the $\zeta-\theta$ phase space is a stable equilibrium point around which resonant electrons rotate with the angular frequency $\omega_{\text {tr }}$, as shown in Fig. 2 . The other point $\left(\zeta_{1}, 0\right)$ is an unstable equilibrium point called a saddle point. The separatrix between trapped and untrapped resonant electrons originates from this saddle point. Eliminating the time variable $t$ from (41) and (42) and integrating them by $\zeta$ and $\theta$, we obtain

$$
\theta^{2}+2 \omega_{\mathrm{tr}}^{2}(\cos \zeta-S \zeta)=C,
$$

which is the equation for trajectories in the $\zeta-\theta$ plane. Substituting the values at the saddle point into (43), we obtain an equation for the separatrix

$$
\theta_{s}= \pm \omega_{\operatorname{tr}} \sqrt{2\left[\cos \zeta_{1}-\cos \zeta+S\left(\zeta-\zeta_{1}\right)\right]}
$$

for $\zeta_{1}<\zeta<\zeta_{2}$, where $\zeta_{2}$ is the phase of the separatrix crossing $\theta=0$. The size of the trapping potential changes as a function of $S(-1 \leq S \leq 1)$. In terms of the parallel velocity $v_{\|}$, electrons near the resonance velocity $V_{R}$ can be trapped and oscillate around $V_{\mathrm{R}}$ with the maximum width of the trapping velocity given by $V_{\mathrm{tr}}=2 \omega_{\mathrm{tr}} / k$ for $S=0$.

The separatrix between the trapped electrons and untrapped electrons plays a critical role in determining the wave growth and damping. The trapped electrons with $S=0$ oscillate around $(\zeta, \theta)=(\pi, 0)$ forming trajectories symmetric around the wave magnetic field. The symmetric trajectories indicate that the trapped electrons received

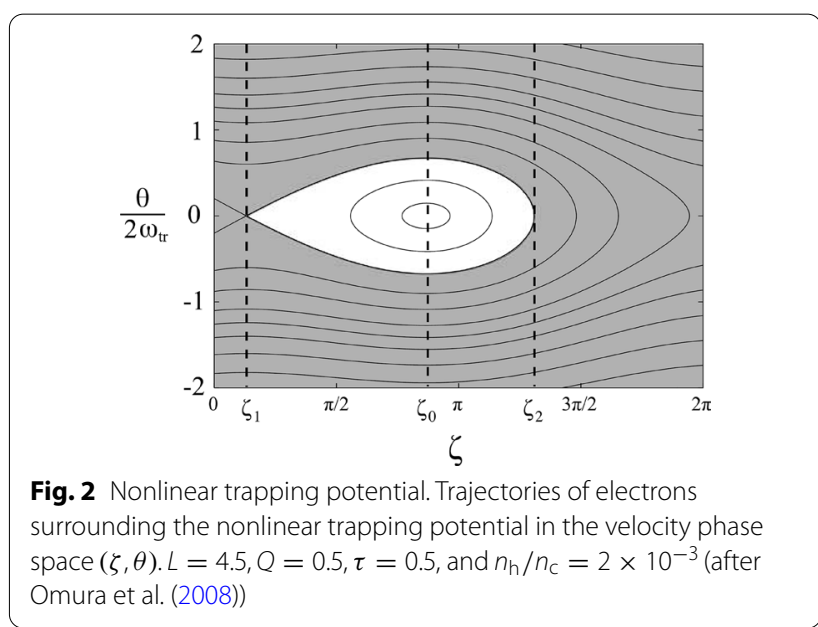


no net energy on average which can be understood by the following equations. The kinetic energy $K$ of an electron is given by

$$
K=m_{0} c^{2}(\gamma-1) .
$$

We can calculate its variation by taking dot products of $v$ and both sides of (11) as

$$
\frac{\mathrm{d} K}{\mathrm{~d} t}=m_{0} \boldsymbol{v} \cdot \frac{\mathrm{d}(\gamma \boldsymbol{v})}{\mathrm{d} t}=-e \boldsymbol{E} \cdot \boldsymbol{v} .
$$

Under the purely transverse electromagnetic wave propagating parallel to the static magnetic field $\boldsymbol{B}_{0}$, we obtain

$$
\frac{\mathrm{d} K}{\mathrm{~d} t}=e E_{\mathrm{w}} \nu_{\perp} \sin \zeta
$$

When resonant electrons trapped by the wave potential are located in the phase range $0<\zeta<\pi$, they are accelerated. Since the center of the trapping potential is given by the second-order resonance condition, we have $\sin \zeta=-S$, and (47) is rewritten for trapped resonant electrons as

$$
\frac{\mathrm{d} K}{\mathrm{~d} t}=-e E_{\mathrm{w}} v_{\perp} S
$$

When chorus emissions with a rising-tone frequency are generated at the equator and propagate toward higher latitudes, we find $S<0$ as we see in (37). Therefore, trapped electrons are accelerated under most of chorus emissions except for falling-tone emissions near the equator, where $S$ can be positive because of a negative sweep rate $\partial \omega / \partial t<0$.

\section{Evolution of wave field due to formation of resonant currents}

\subsection{Distribution functions of energetic electrons}

As the velocity distribution function of energetic electrons, we often assume a bi-Maxwellian with Gaussian functions of velocity components $v_{\|}$and $v_{\perp}$ which go to $\pm \infty$. When we consider the relativistic energy range, the definition of the velocity distribution as the bi-Maxwellian becomes inconvenient because of the limitation by the speed of light $c$. To avoid the inconvenience, we use a momentum defined by $u=\gamma v$ in place of a velocity $v$. The range of the momentum is not limited, while the dimension is the same as the velocity $v$ because the Lorentz factor $\gamma=\left(1-c^{2} / v^{2}\right)^{-1 / 2}$ is dimensionless. To implement the loss cone distribution of energetic electrons trapped by the Earth's dipole magnetic field, we assume a subtracted Maxwellian distribution function at the equator given by

$$
\begin{aligned}
f\left(u_{\|}, u_{\perp}\right)= & \frac{N_{0}}{(2 \pi)^{3 / 2} U_{t \|} U_{t \perp}^{2}(1-\rho \beta)} \exp \left(-\frac{u_{\|}^{2}}{2 U_{t \|}^{2}}\right) \\
& \cdot\left[\exp \left(-\frac{u_{\perp}^{2}}{2 U_{t \perp}^{2}}\right)-\rho \exp \left(-\frac{u_{\perp}^{2}}{2 \beta U_{t \perp}^{2}}\right)\right],
\end{aligned}
$$

where $\rho(0 \leq \rho \leq 1)$ and $\beta(0<\beta<1)$ specify relative height and width of a momentum distribution subtracted from a Maxwellian distribution, respectively. With $\rho=1$, a complete loss cone is realized. Summers et al. (2012) showed that a bi-Maxwellian distribution function at the equator can keep the shape of the bi-Maxwellian distribution function at a distance away from the equator. Therefore, we assume a subtracted Maxwellian distribution function as a distance $h$ from the equator as

$$
\begin{aligned}
f_{h}\left(u_{\| h}, u_{\perp h}\right)= & \frac{N_{h}}{(2 \pi)^{3 / 2} U_{t \| h} U_{t \perp h}^{2}\left(1-\rho \beta_{h}\right)} \exp \left(-\frac{u_{\| h}^{2}}{2 U_{t \| h}^{2}}\right) \\
& \cdot\left[\exp \left(-\frac{u_{\perp h}^{2}}{2 U_{t \perp h}^{2}}\right)-\rho \exp \left(-\frac{u_{\perp h}^{2}}{2 \beta_{h} U_{t \perp h}^{2}}\right)\right] .
\end{aligned}
$$

From Liouville's theorem, we have

$$
f_{h}\left(u_{\| h}, u_{\perp h}\right)=f\left[u_{\|}\left(u_{\| h}, u_{\perp h}, h\right), u_{\perp}\left(u_{\| h}, u_{\perp h}, h\right)\right] .
$$

Preservation of the first adiabatic invariant and energy conservation of an electron give

$$
\begin{aligned}
& u_{\perp}^{2}=\frac{B_{0}}{B_{h}} u_{\perp h}^{2}, \\
& u_{\|}^{2}=u_{\| h}^{2}+\left(1-\frac{B_{0}}{B_{h}}\right) u_{\perp h}^{2} .
\end{aligned}
$$

Substituting (52) and (53) into (51), we obtain

$$
\begin{gathered}
f_{h}\left(u_{\| h}, u_{\perp h}\right)=\frac{N_{0}}{(2 \pi)^{3 / 2} U_{t \|} U_{t \perp}^{2}(1-\rho \beta)} \exp \left(-\frac{u_{\| h}^{2}}{2 U_{t \|}^{2}}\right) \\
\cdot\left\{\exp \left[-\left(\frac{1-B_{0} / B_{h}}{2 B_{h} U_{t \|}^{2}}+\frac{B_{0}}{2 B_{h} U_{t \perp}^{2}}\right) u_{\perp h}^{2}\right]\right. \\
\left.-\exp \left[-\left(\frac{1-B_{0} / B_{h}}{2 B_{h} U_{t \|}^{2}}+\frac{B_{0}}{2 \beta B_{h} U_{t \perp}^{2}}\right) u_{\perp h}^{2}\right]\right\} .
\end{gathered}
$$

Comparing (50) and (54), and assuming $B_{h}=B_{0}\left(1+a h^{2}\right)$, we have

$$
\begin{aligned}
& U_{t \| h}=U_{t \|}, \\
& U_{t \perp h}=W_{h} U_{t \perp},
\end{aligned}
$$




$$
\begin{aligned}
& \beta_{h}=\left[1+\frac{W_{h}^{2}}{1+a h^{2}}\left(\frac{1}{\beta}-1\right)\right]^{-1}, \\
& N_{h}=W_{h}^{2} \frac{1-\rho \beta_{h}}{1-\rho \beta} N_{0},
\end{aligned}
$$

where

$$
W_{h}=\left(1+\frac{a h^{2}}{1+a h^{2}} A_{0}\right)^{-1 / 2},
$$

and

$$
A_{0}=\frac{U_{t \perp}^{2}}{U_{t \|}^{2}}-1
$$

For the analysis of the nonlinear trapping and associated wave growth at a distance $h$, a simplified distribution function $f_{\mathrm{t}}\left(u_{\|}, u_{\perp}\right)$ with the following form is assumed.

$$
f_{\mathrm{t}}\left(u_{\|}, u_{\perp}\right)=K \exp \left(-\frac{u_{\|}^{2}}{2 U_{t \| h}^{2}}\right) \delta\left(u_{\perp}-U_{\perp h}\right) .
$$

Assuming the total density of the energetic electrons is the same with the two distribution functions, we have

$$
\int f_{\mathrm{t}}\left(u_{\|}, u_{\perp}\right) 2 \pi u_{\perp} \mathrm{d} u_{\perp} \mathrm{d} u_{\|}=\int f\left(u_{\|}, u_{\perp}\right) 2 \pi u_{\perp} \mathrm{d} u_{\perp} \mathrm{d} u_{\|}=N_{h} .
$$

Substituting (61) into (62), we obtain

$$
K=\frac{N_{h}}{(2 \pi)^{3 / 2} U_{t \| h} U_{\perp h}} .
$$

We also assume the equality of the perpendicular momentum

$$
\int u_{\perp} f_{\mathrm{t}}\left(u_{\|}, u_{\perp}\right) 2 \pi u_{\perp} \mathrm{d} u_{\perp} \mathrm{d} u_{\|}=\int u_{\perp} f\left(u_{\|}, u_{\perp}\right) 2 \pi u_{\perp} \mathrm{d} u_{\perp} \mathrm{d} u_{\|} .
$$

Substituting (50) and (61) into (64), we obtain

$$
U_{\perp h}=\left(\frac{\pi}{2}\right)^{1 / 2} \frac{1-\rho \beta_{h}^{3 / 2}}{1-\rho \beta_{h}} U_{t \perp} .
$$

Using (65), (63), and (58), we can rewrite (61) as

$$
f_{\mathrm{t}}\left(u_{\|}, u_{\perp}\right)=\frac{N_{h}}{(2 \pi)^{3 / 2} U_{t \| h} U_{\perp h}} \exp \left(-\frac{u_{\|}^{2}}{2 U_{t \| h}}\right) \delta\left(u_{\perp}-U_{\perp h}\right) .
$$

\subsection{Resonant currents and wave evolution}

Resonant electrons $v_{\|} \sim V_{\mathrm{R}}$ are divided into two groups. One is trapped resonant electrons inside the nonlinear wave potential described above. The other is those outside the nonlinear potential. The shape of the trapping potential in $\zeta-\theta$ phase space changes as a function of $S$ as we have seen above. Trajectories of trapped and untrapped resonant electrons become very different because of the variation of the resonance velocity, by which the trapped electrons are guided, while the untrapped electrons follow adiabatic motion except for the moment crossing the resonance velocity. The difference in the number densities of the trapped and untrapped resonant electrons give rise to a resonant current $J_{R}$, which is decomposed into $J_{B}$ and $J_{\mathrm{E}}$ parallel to the wave magnetic field and electric field, respectively. These currents are calculated by

$$
\begin{aligned}
& J_{\mathrm{B}}=\int_{0}^{\infty} \int_{0}^{2 \pi} \int_{-\infty}^{\infty}\left[-e v_{\perp} \cos \zeta\right] f\left(u_{\|}, \zeta, u_{\perp}\right) u_{\perp} \mathrm{d} u_{\|} \mathrm{d} \zeta \mathrm{d} u_{\perp}, \\
& J_{\mathrm{E}}=\int_{0}^{\infty} \int_{0}^{2 \pi} \int_{-\infty}^{\infty}\left[e v_{\perp} \sin \zeta\right] f\left(u_{\|}, \zeta, u_{\perp}\right) u_{\perp} \mathrm{d} u_{\|} \mathrm{d} \zeta \mathrm{d} u_{\perp},
\end{aligned}
$$

where $f\left(u_{\|}, \zeta, u_{\perp}\right)$ is the momentum distribution function of energetic electrons representing the phase space density in the three-dimensional momentum space. The cold electrons supporting the wave propagation is not included in the distribution.

From Maxwell's equations and the equations of motion of cold and energetic electrons, we can obtain a set of equations describing the evolution of electromagnetic wave field (Omura et al. 2008).

$$
\begin{aligned}
& \frac{\partial B_{\mathrm{w}}}{\partial t}+V_{\mathrm{g}} \frac{\partial B_{\mathrm{w}}}{\partial h}=-\frac{\mu_{0} V_{\mathrm{g}}}{2} J_{\mathrm{E}}, \\
& c^{2} k^{2}-\omega^{2}-\frac{\omega \omega_{\mathrm{pe}}^{2}}{\Omega_{e}-\omega}=\mu_{0} c^{2} k \frac{J_{\mathrm{B}}}{B_{\mathrm{w}}},
\end{aligned}
$$

where $\mu_{0}$ is the magnetic permeability in vacuum. The resonant current $J_{\mathrm{E}}$ contributes to the variation of the wave amplitude, i.e., wave growth or damping, while $J_{\mathrm{B}}$ changes the dispersion relation of the wave as a nonlinear term that changes the wave frequency. These resonant currents are initially formed by a triggering wave packet with the frequency $\omega_{0}$ and the wave number $k$ which satisfy (3). Namely, we have

$$
c^{2} k^{2}=\omega_{0}^{2}+\frac{\omega_{0} \omega_{\mathrm{pe}}^{2}}{\Omega_{\mathrm{e}}-\omega_{0}} .
$$

Since the spatial structure of the wave phase is imposed by the wave packet of the triggering wave, the wave number $k$ or the wavelength does not change in a short time scale, while the rate of the wave phase variation in time or the wave frequency changes in the presence of 
$J_{\mathrm{B}}$. Denoting the frequency deviation from $\omega_{0}$ as $\delta \omega$, i.e., $\omega=\omega_{0}+\delta \omega$ and assuming $\delta \omega \ll \omega_{0}$, we expand (70) around $\omega_{0}$ to obtain

$$
\left\{2 \omega_{0}+\frac{\Omega_{\mathrm{e}} \omega_{\mathrm{pe}}^{2}}{\left(\Omega_{\mathrm{e}}-\omega_{0}\right)^{2}}\right\} \delta \omega=-\mu_{0} c^{2} k \frac{J_{\mathrm{B}}}{B_{\mathrm{w}}} .
$$

Differentiating (71) with respect to $\omega_{0}$, we have

$$
2 c^{2} k \frac{\partial k}{\partial \omega_{0}}=2 \omega_{0}+\frac{\Omega_{\mathrm{e}} \omega_{\mathrm{pe}}^{2}}{\left(\Omega_{\mathrm{e}}-\omega_{0}\right)^{2}} .
$$

From (72) and (73), we obtain

$$
\delta \omega=-\frac{\mu_{0} V_{\mathrm{g}}}{2} \frac{J_{\mathrm{B}}}{B_{\mathrm{w}}} .
$$

As we have analyzed the nonlinear motion of resonant electrons, the magnitude of the perpendicular velocity controls the width of the trapping potential and the period of the trapping motion. For simplicity of the analysis, we integrate the distribution function in $u_{\perp}$ in the calculation of the resonant currents by assuming the distribution is expressed by the following form:

$$
f\left(u_{\|}, \zeta, u_{\perp}\right)=g\left(u_{\|}, \zeta\right) p\left(u_{\perp}\right) .
$$

The average perpendicular momentum $U_{\perp 0}$ is calculated by

$$
U_{\perp 0}=\frac{\int_{0}^{\infty} u_{\perp} p\left(u_{\perp}\right) 2 \pi u_{\perp} \mathrm{d} u_{\perp}}{\int_{0}^{\infty} p\left(u_{\perp}\right) 2 \pi u_{\perp} \mathrm{d} u_{\perp}} .
$$

Substituting (75) into (68), and replacing $p\left(u_{\perp}\right)$ with a Dirac delta function $\delta\left(u_{\perp}-U_{\perp 0}\right)$, we obtain

$$
J_{\mathrm{E}}=e \gamma^{-1} U_{\perp 0}^{2} \int_{0}^{2 \pi} \int_{-\infty}^{\infty} g\left(u_{\|}, \zeta\right) \sin \zeta \mathrm{d} u_{\|} \mathrm{d} \zeta .
$$

To realize a loss cone distribution function, we often assume a subtracted Maxwellian distribution function given by

$$
p\left(u_{\perp}\right)=\frac{1}{1-\rho \beta}\left[\exp \left(-\frac{u_{\perp}^{2}}{2 U_{t \perp}^{2}}\right)-\rho \exp \left(-\frac{u_{\perp}^{2}}{2 \beta U_{t \perp}^{2}}\right)\right] .
$$

The average perpendicular momentum $U_{\perp 0}$ is obtained from (76) as

$$
U_{\perp 0}=\sqrt{\frac{\pi}{2}}\left(\frac{1-\rho \beta^{3 / 2}}{1-\rho \beta}\right) U_{t \perp} .
$$

Under the assumption that $u_{\perp} \sim U_{\perp 0}$, formation of the resonance current $J_{\mathrm{E}}$ and $J_{\mathrm{B}}$ is described by the structure of $g\left(u_{\|}, \zeta\right)$. Since the dynamics of trapped resonant electrons is much different from that of untrapped electrons, there occurs a distinct difference in the distribution of trapped electrons. Representing the initial distribution of trapped electrons by $g_{t}\left(u_{\|}, \zeta\right)$, we express the total distribution function of resonant electrons by

$$
g\left(u_{\|}, \zeta\right)=g_{0}\left(u_{\|}\right)-Q g_{t}\left(u_{\|}, \zeta\right),
$$

where $g_{0}\left(u_{\|}\right)$is a unperturbed distribution function, and $Q$ is the depth of an electron hole due to depletion of trapped resonant electrons in the velocity phase space. Assuming that $g_{t}\left(u_{\|}, \zeta\right)=G$ inside the trapping region and that $g_{t}\left(u_{\|}, \zeta\right)=0$ outside the trapping region, we rewrite (77) as

$$
J_{\mathrm{E}}=-J_{0} \int_{\zeta_{1}}^{\zeta_{2}}\left[\cos \zeta_{1}-\cos \zeta+S\left(\zeta-\zeta_{1}\right)\right]^{1 / 2} \sin \zeta \mathrm{d} \zeta .
$$

Similarly we obtain

$$
J_{\mathrm{B}}=J_{0} \int_{\zeta_{1}}^{\zeta_{2}}\left[\cos \zeta_{1}-\cos \zeta+S\left(\zeta-\zeta_{1}\right)\right]^{1 / 2} \cos \zeta \mathrm{d} \zeta,
$$

where

$$
J_{0}=(2 e)^{3 / 2}\left(m_{0} k\right)^{-1 / 2} \gamma^{-1} \chi Q G U_{\perp 0}^{5 / 2} B_{\mathrm{w}}^{1 / 2} .
$$

The constants $e$ and $m_{0}$ are the absolute value of charge and the rest mass of an electron, respectively. The expression of $J_{0}$ is slightly different from that in Omura et al. (2008). This is because we have assumed the distribution function (75) in momentum rather than in velocity. The value $G$ in Omura et al. (2008) is in velocity, while the same $G$ is used as in momentum in Omura et al. (2009) in deriving the nonlinear growth rate and the optimum and threshold wave amplitudes, which resulted in different powers of the Lorentz factor $\gamma$ in these expressions. The nonlinear growth rates and the threshold wave amplitude are derived consistently based on the momentum distribution function in the followings.

In evaluating $G$, we assume the simplified momentum distribution function (66) at the magnetic equator as

$$
f\left(u_{\|}, u_{\perp}\right)=\frac{N_{0}}{(2 \pi)^{3 / 2} U_{t \|} U_{\perp 0}} \exp \left(-\frac{u_{\|}^{2}}{2 U_{t \|}^{2}}\right) \delta\left(u_{\perp}-U_{\perp 0}\right),
$$

where $U_{\perp 0}=\gamma V_{\perp 0}$, and $U_{t \|}$ is the thermal momentum in the parallel direction. We have normalized the distribution to the density of hot electrons $N_{0}$ at the magnetic equator. Integrating $f$ over $u_{\perp}$, we obtain $G$ of the unperturbed distribution function $g_{0}\left(u_{\|}\right)$at the resonance velocity $V_{\mathrm{R}}$ as 


$$
G=\frac{N_{0}}{(2 \pi)^{3 / 2} U_{t \|} U_{\perp 0}} \exp \left(-\frac{\gamma^{2} V_{\mathrm{R}}^{2}}{2 U_{t \|}^{2}}\right) .
$$

To evaluate $G$ at a distance $h$ from the equator, we replace $N_{0}$ with $N_{h}$ given by (58) and use $U_{\perp h}$ given by (65) in place of $U_{\perp 0}$, respectively. It should be noted that $U_{t \perp}$ and $\beta$ vary as functions of $h$ as denoted by $U_{t \perp h}$ and $\beta_{h}$ in (56) and (57), respectively, while $U_{t \|}$ is a constant as indicated by (55).

We evaluate the integrals in (81) and (82) numerically, and we plot the normalized currents $-J_{\mathrm{E}} / J_{0}$ and $-J_{\mathrm{B}} / J_{0}$ as functions of $S$ for $-1<S<0$ in Fig. 3. The maximum value of $-J_{\mathrm{E}} / J_{0}$ is 0.975 at $S=-0.413$, which gives $J_{\mathrm{B}}=-1.3 J_{0}$. Since the negative $J_{\mathrm{E}}$ causes wave growth, we can expect the maximum wave growth at $S=-0.4$, which can be realized at the equator when we have the frequency increase as indicated in (37). Since the negative $J_{\mathrm{B}}$ causes a frequency increase, as shown in (74), we can assume an optimum condition for the nonlinear wave growth. Namely, when the frequency increase $\delta \omega$ takes place because of gradual formation of $J_{\mathrm{B}}$ over a time $T_{\mathrm{N}}$, we have a frequency sweep rate on average specified by

$$
\frac{\partial \omega}{\partial t}=\frac{\delta \omega}{T_{\mathrm{N}}} .
$$

We call the time $T_{\mathrm{N}}$ as the nonlinear transition time, and compare it with the nonlinear trapping time $T_{\text {tr }}$ by introducing a parameter $\tau=T_{\mathrm{N}} / T_{\mathrm{tr}}$, where the nonlinear trapping time is given by

$$
T_{\mathrm{tr}}=\frac{2 \pi}{\omega_{\mathrm{tr}}}=\frac{2 \pi}{\chi}\left(\frac{m_{0} \gamma}{k V_{\perp 0} e B_{\mathrm{w}}}\right)^{1 / 2} .
$$

Setting $\partial \Omega_{\mathrm{e}} / \partial h=0$ and $S=-0.4$ in (37), we have the optimum frequency sweep rate for the nonlinear wave growth at the equator as

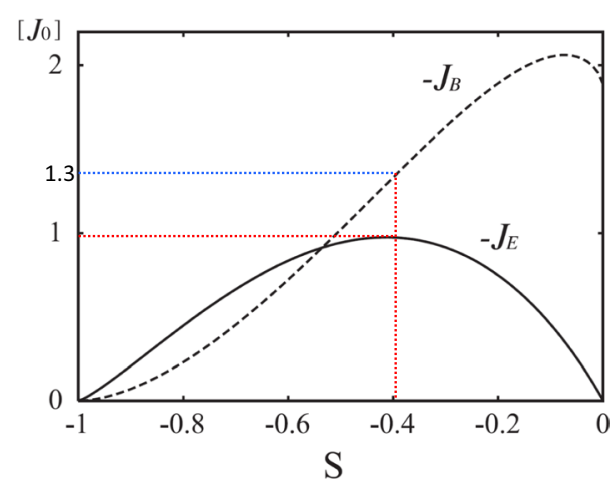

Fig. 3 Resonant currents as function os $S$. Variation of resonant currents $J_{E}$ in solid line and $J_{B}$ in dashed line as functions of the inhomogeneity factor $S$. At $S=-0.413, J_{E}=-0.975 J_{0}$ and $J_{B}=-1.3 J_{0}$ as indicated by dotted lines

$$
\frac{\partial \omega}{\partial t}=\frac{0.4 s_{0} \omega}{s_{1}} \Omega_{\mathrm{w}}
$$

The relation between the frequency sweep rate $\partial \omega / \partial t$ and the wave amplitude $\Omega_{\mathrm{w}}(88)$ has been confirmed by the simulation shown in Fig. 5 and observations (Kurita et al. 2012; Foster et al. 2017).

\subsection{Role of linear growth rates}

To initiate the nonlinear wave growth process, we need a triggering wave with a finite amplitude greater than the threshold amplitude for nonlinear wave growth. The triggering wave can be generated naturally from the thermal fluctuation of electromagnetic field if the linear growth rates of whistler mode waves are positive in the presence of energetic electrons. We assume a subtracted Maxwellian distribution function of the energetic electrons given by (49). With non-relativistic electrons, the parallel and perpendicular components $U_{t \|}$ and $U_{t \perp}$ of thermal momentum can be regarded as the parallel and perpendicular components of thermal velocity as defined in the dispersion solver KUPDAP (Sugiyama et al. 2015). With a momentum distribution function of subtracted Maxwellian distribution function including the bi-Maxwellian distribution function as a special case of $\rho=0$ with a temperature anisotropy $U_{t \perp}>U_{t \|}$, we find the linear growth rate becomes positive over a range of frequency and corresponding wave number in the quasi-parallel direction with its maximum value with the wave number vector purely parallel to the background magnetic field, as shown in Fig. 4. We assumed a typical plasma frequency as $\omega_{\mathrm{pe}}=4 \Omega_{\mathrm{ce}}$. With energetic electrons higher than $30 \mathrm{keV}$, the linear growth rate $\Gamma_{\mathrm{L}}$ takes positive values only in the range below half the cyclotron frequency. In the presence of the temperature anisotropy, unstable wave modes grow from the thermal fluctuation level. Waves near the maximum linear growth rates grow with the linear growth rates initially. The mode with the maximum linear growth rate forms a coherent wave attaining a largest wave amplitude, and it suppresses the growth of adjacent wave modes. The coherent wave becomes a triggering wave for the nonlinear wave growth process.

\subsection{Nonlinear growth rate}

The nonlinear wave growth is due to the formation of resonant currents through phase organization of resonant electrons in the presence of nonlinear trapping potential of a coherent triggering wave. The potential is formed by the Lorentz force $-e \boldsymbol{V}_{\perp 0} \times \boldsymbol{B}_{\mathrm{w}}$ acting on electrons with parallel velocities close to the cyclotron resonance velocity $V_{\mathrm{R}}$. Although a large $U_{\perp 0}$ makes the trapping potential large, the temperature anisotropy of energetic electrons 

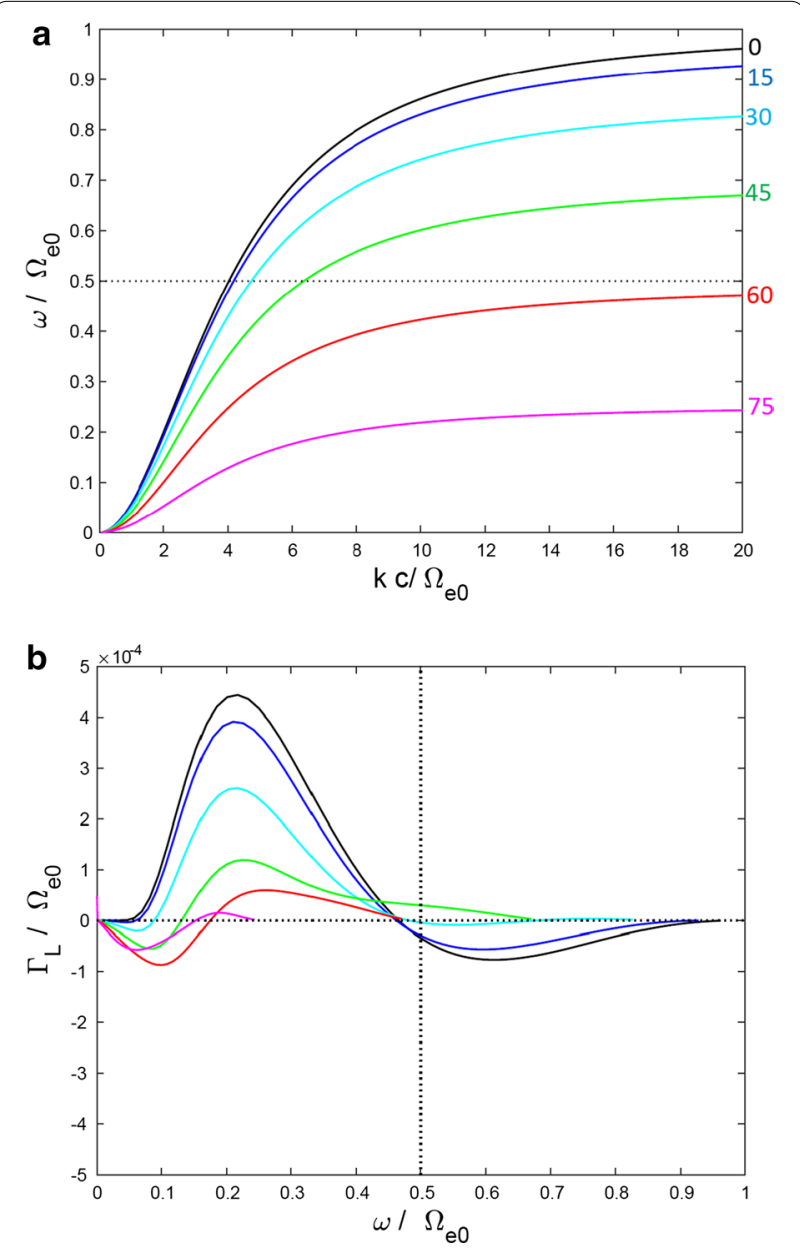

Fig. 4 Linear dispersion relation. Linear dispersion relation of a plasma consisting of cold electrons, cold ions, and minor hot electrons $\left(n_{h} / n_{c}=2 \times 10^{-3}\right)$. A subtracted Maxwellian for the energetic electrons is assumed with $\beta=0.3, \rho=1.0, U_{t \|}=0.25 c$, and $U_{t \perp}=3.0 \mathrm{c}$. The frequencies and linear growth rates for different wave normal directions $\theta=0,15,30,45,60,75$ degrees are shown in the same colors, respectively

is not directly required for the nonlinear wave growth. This is very different from the linear growth rate which requires the temperature anisotropy $T_{\perp}>T_{\|}$. Even with a condition of the negative linear growth rate, we can have nonlinear wave growth in the presence of large amplitude wave. The source of energy for the nonlinear growth comes from the perpendicular kinetic energy of resonant electrons as expressed by (68) for $J_{\mathrm{E}}$. Under a coherent triggering wave, resonant electrons are organized in gyrophase $\zeta$, resulting in a negative $J_{\mathrm{E}}$. Because $\boldsymbol{J}_{\mathrm{R}} \cdot \boldsymbol{E}_{\mathrm{w}}<0$, the transfer of energy from the resonant electrons to the wave field takes place.

We define the nonlinear growth rate based on the wave equation (69) describing the evolution of the wave amplitude. In a frame of reference moving with the group velocity $V_{\mathrm{g}},(69)$ is rewritten as

$$
\frac{\mathrm{d} B_{\mathrm{w}}}{\mathrm{d} t}=\Gamma_{\mathrm{N}} B_{\mathrm{w}}
$$

where

$$
\Gamma_{\mathrm{N}}=-\frac{\mu_{0} V_{\mathrm{g}}}{2} \frac{J_{\mathrm{E}}}{B_{\mathrm{w}}} .
$$

Assuming an electron hole shown in Fig. 2, we can find the maximum value of $-J_{\mathrm{E}}$ with $S=-0.4$. Since $-J_{\mathrm{E}} / J_{0}=0.975 \sim 1$, we have from (83)

$$
J_{\mathrm{E}, \max }=-(2 e)^{3 / 2}\left(m_{0} k\right)^{-1 / 2} \gamma^{-1} \chi Q G U_{\perp 0}^{5 / 2} B_{\mathrm{w}}^{1 / 2} .
$$

Substituting $J_{\mathrm{E}, \max }$ with $J_{\mathrm{E}}$ in (90), and using (85) for the distribution function (84), we obtain the nonlinear growth rate

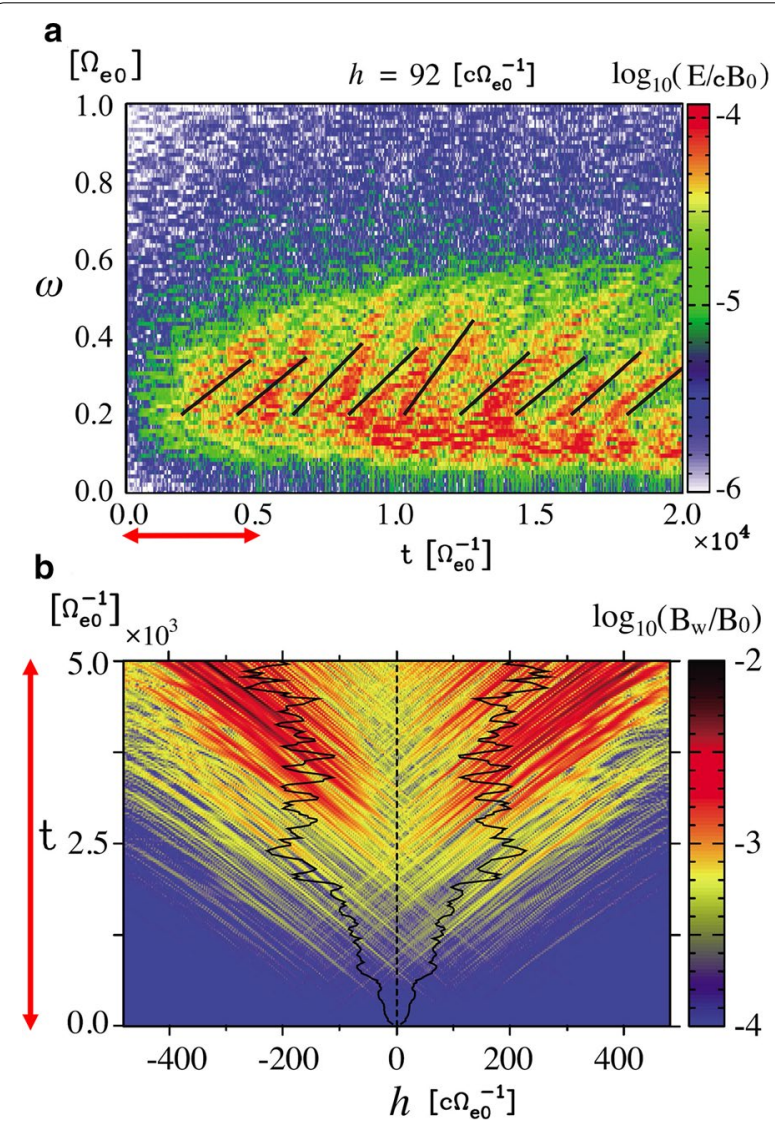

Fig. 5 Chorus simulation. Electron hybrid simulation of whistler-mode chorus emissions. a Dynamics spectra of transverse electric field. $\mathbf{b}$ Spatial and temporal evolution of wave amplitudes $B_{\mathrm{w}}$ of chorus emissions in the early period of the simulation run indicated by red arrows. Solid lines show location of the critical distance $\pm h_{c}(t)$ from the equator, where $h_{c}(t)$ is evaluated from the wave amplitude $B_{\mathrm{w}}(h, t)$ averaged over $h=-10 \sim 10 c \Omega_{\mathrm{e} 0}^{-1}$ (after Katoh and Omura (2011)) 


$$
\Gamma_{\mathrm{N}}=\frac{Q \omega_{\mathrm{ph}}^{2} V_{\mathrm{g}}}{2 \gamma U_{t \|}}\left(\frac{\xi}{\omega \Omega_{\mathrm{w}}}\right)^{1 / 2}\left(\frac{\chi U_{\perp h}}{\pi c}\right)^{3 / 2} \exp \left(-\frac{\gamma^{2} V_{\mathrm{R}}^{2}}{2 U_{t \|}^{2}}\right) .
$$

The parameter $\omega_{\mathrm{ph}}$ is the plasma frequency of hot electrons given by $\omega_{\mathrm{ph}}^{2}=N_{h} e^{2} /\left(m_{0} \epsilon_{0}\right)$, where $\epsilon_{0}$ is the vacuum permittivity, and $N_{h}$ and $U_{\perp h}$ are functions of $h$ evaluated by $(55) \sim(60)$ and (65). The nonlinear growth rate is evaluated at a distance $h$ from the equator by (92). The Lorentz factor $\gamma$ is calculated for the trapped resonant electrons from (12) with $v_{\|}=V_{\mathrm{R}}$ and $v_{\perp}=U_{\perp h} / \gamma$.
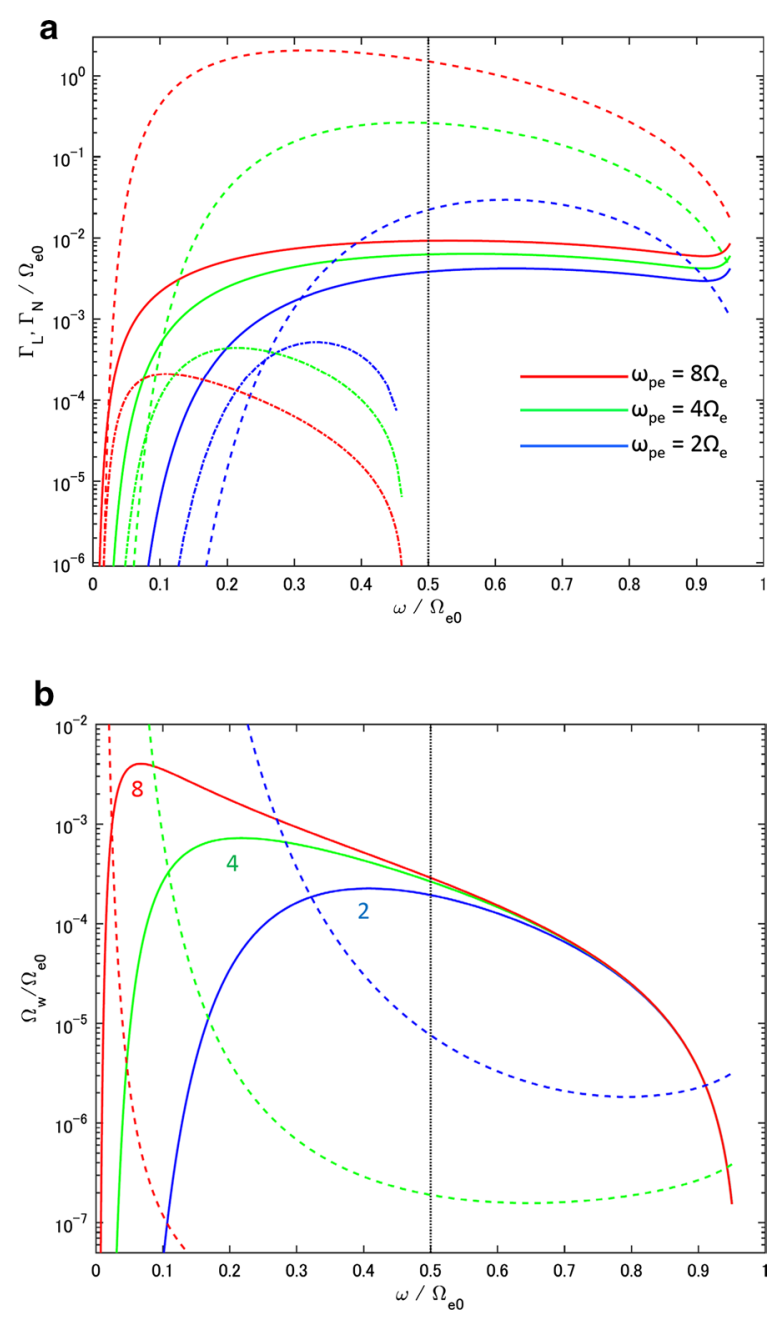

Fig. 6 Nonlinear wave growth theory. a Linear growth rates (dashdot lines) and nonlinear growth rates at the threshold amplitudes (dashed lines) and the nonlinear growth rates at the optimum amplitudes (solid lines). A subtracted Maxwellian for the energetic electrons is assumed with $\beta=0.3, \rho=1.0, U_{t \|}=0.25 c$, and $U_{t \perp}=3.0 \mathrm{c}$. $\mathbf{b}$ Optimum and threshold amplitudes in solid and dashed lines for different values of the plasma frequency. $\omega_{\text {pe }}=2$, $4,8 \Omega_{\mathrm{e}}$ in blue, green, and red, respectively. The plasma frequency of hot electrons is kept constant as $\omega_{\text {ph }}=0.1789$ corresponding to $n_{\mathrm{h}} / n_{\mathrm{c}}=2 \times 10^{-3}$ for $\omega_{\mathrm{pe}}=4 \Omega_{\mathrm{e}}$
The nonlinear growth rate is a function of the wave amplitude $\Omega_{\mathrm{w}}\left(=e B_{\mathrm{w}} / m_{0}\right)$, while the linear growth rate is a constant for a specific set of parameters regardless of the wave amplitude. In Fig. 6a, both linear and nonlinear growth rates are plotted for three different cold plasma densities as specified by the plasma frequencies $\omega_{\mathrm{pe}} / \Omega_{\mathrm{e}}=2,4,8$, while the density of energetic electrons is assumed to be constant as $\omega_{\mathrm{ph}}=0.1789$ corresponding to $n_{\mathrm{h}} / n_{\mathrm{c}}=2 \times 10^{-3}$ in the case of $\omega_{\mathrm{pe}}=4 \Omega_{\mathrm{e}}$. The energetic electrons form a subtracted Maxwellian distribution functions given by (49) with $\beta=0.3, \rho=1.0, U_{t \|}=0.25 c$, and $U_{t \perp}=0.3 c$. The nonlinear growth rates are calculated for optimum amplitudes in solid lines and for threshold amplitudes in dashed lines, which are plotted in Fig. 6a. Derivations of threshold and optimum amplitudes are given in the following subsections. The linear growth rates plotted in dash-dot lines are much smaller than the nonlinear growth rates. Peaks in both linear and nonlinear growth rates shift to the lower frequency ranges with higher plasma densities.

\subsection{Absolute instability}

As we have seen in (10), the frequency of the wave packet is constant in the frame of reference moving with the group velocity. The frequency only changes near the equator where we can have large $-J_{\mathrm{B}} / B_{\mathrm{W}}$ inducing the frequency deviation $\delta \omega$ given by (72). The wave amplitude $B_{\mathrm{w}}$ should increase to form a new wave packet. Expressing the derivative $\mathrm{d} B_{\mathrm{w}} / \mathrm{d} t$ in (89) in terms of temporal and spatial derivatives and normalizing the wave amplitude, we have

$$
\frac{\partial \Omega_{\mathrm{w}}}{\partial t}+V_{\mathrm{g}} \frac{\partial \Omega_{\mathrm{w}}}{\partial h}=\Gamma_{\mathrm{N}} \Omega_{\mathrm{w}}
$$

To have the wave growth locally, i.e., an absolute instability, we need $\partial \Omega_{\mathrm{w}} / \partial t>0$. We obtain from (93)

$$
\frac{\Gamma_{\mathrm{N}}}{V_{\mathrm{g}}} \Omega_{\mathrm{w}}>\frac{\partial \Omega_{\mathrm{w}}}{\partial h},
$$

where we have assumed that the chorus wave packet propagates in the positive $h$ direction, i.e., $V_{\mathrm{g}}>0$.

Frequency variation is only possible at the time of localized wave generation before the wave number structure in space is formed over a distance much greater than a spatial scale of the nonlinear resonant current. Once the wave number structure is given it becomes difficult to change the frequency from the value determined by the cold plasma dispersion relation. Therefore, the chorus emission with substantial frequency variation is only possible by the localized absolute instability rather than the convective instability. 


\subsection{Optimum wave amplitude}

As the wave grows at a frequency of the largest linear growth rate, the wave becomes coherent suppressing the growth of other waves around the frequency. Once the wave amplitude exceeds a threshold amplitude for an absolute nonlinear instability, the wave amplitude grows with frequency increasing monotonically at the equator (Omura et al. 2009). The nonlinear wave growth stops near the optimum wave amplitude (Omura and Nunn 2011) and then decreases gradually to the level of the threshold amplitude, resulting in a short subpacket of a chorus wave element.

We evaluate $J_{\mathrm{B}}$ expressed by (82) with $\mathrm{S}=-0.4$ for the maximum $J_{\mathrm{E}}$, which gives $J_{\mathrm{B}}=-1.3 J_{0}$, as shown in Fig. 3 . Namely, we have

$$
J_{\mathrm{B}}=-1.3(2 e)^{3 / 2}\left(m_{0} k\right)^{-1 / 2} \gamma^{-1} \chi Q G U_{\perp 0}^{5 / 2} B_{\mathrm{w}}^{1 / 2} .
$$

Substituting (95) into (74), and using (85) and (87), we calculate the frequency sweep rate $\delta \omega / T_{\mathrm{N}}$ due to formation of $J_{\mathrm{B}}$ over the nonlinear transition time given by (87).

$$
\frac{\delta \omega}{T_{\mathrm{N}}}=\frac{1.3 Q V_{\mathrm{g}}}{4 \tau U_{t \|}} \pi^{-5 / 2}\left(\frac{\omega_{\mathrm{ph}} U_{\perp 0} \chi}{\gamma c}\right)^{2} \exp \left(-\frac{\gamma^{2} V_{\mathrm{R}}^{2}}{2 U_{t \|}^{2}}\right) .
$$

Equating (86) and (88), we obtain an amplitude at which the optimum condition for nonlinear wave growth is satisfied. Solving for $B_{\mathrm{w}}$, we obtain the normalized optimum wave amplitude $\tilde{\Omega}_{\text {op }}$ as

$$
\begin{aligned}
\tilde{\Omega}_{\mathrm{op}}= & 0.8 \pi^{-5 / 2} \frac{|Q| \tilde{V}_{\mathrm{p}} \tilde{V}_{\mathrm{g}}}{\tau \tilde{\omega}} \frac{\tilde{U}_{\perp 0}}{\tilde{U}_{t \|}} \tilde{\omega}_{\mathrm{ph}}^{2} \\
& \cdot\left(1-\frac{\tilde{V}_{\mathrm{R}}}{\tilde{V}_{\mathrm{g}}}\right)^{2} \exp \left(-\frac{\gamma^{2} \tilde{V}_{\mathrm{R}}^{2}}{2 \tilde{U}_{t \|}^{2}}\right),
\end{aligned}
$$

where $\quad \tilde{\Omega}_{\mathrm{op}}=\Omega_{\mathrm{op}} / \Omega_{\mathrm{e} 0}=B_{\mathrm{w}} / B_{0}, \quad \tilde{\omega}_{\mathrm{ph}}=\omega_{\mathrm{ph}} / \Omega_{\mathrm{e} 0}$, $\tilde{U}_{t \|}=U_{t \|} / c$, and $\tilde{V}_{\mathrm{p}}=V_{\mathrm{p}} / c=\chi \xi$. We can apply the same logic to derive the optimum amplitude for the nonlinear wave growth due to an enhancement of trapped resonant electrons forming a positive $J_{\mathrm{B}}$ producing a falling tone emission (Omura et al. 2015a). We represent an electron enhancement forming an electron hill by a negative value of $Q$. Therefore, we use the absolute value of $Q$ in (97).

Using the optimum wave amplitude, we can rewrite the nonlinear transition time in a normalized form

$$
T_{\mathrm{N}} \Omega_{\mathrm{e} 0}=2 \pi \gamma \tau\left(\frac{\xi}{\chi \tilde{U}_{\perp 0} \tilde{\omega} \tilde{\Omega}_{\mathrm{op}}}\right)^{1 / 2}
$$

Over the period of $T_{\mathrm{N}}$ a subpacket grows and then damps out over nearly the same period of $T_{\mathrm{N}}$. The subpacket propagates away from the equator interacting with counter streaming resonant electrons in the downstream of the wave propagation. The gyro-phases of the resonant electrons are modulated by the wave with frequencies higher than that of the original triggering wave. The phase-modulated resonant electrons carry the information of the new wave packet by forming spatial structure with wavenumber $k$ of the newly generated wave. The electrons move upstream, and generate a new triggering wave with the higher frequency in the upstream from the equator. The new wave triggers another cycle of the nonlinear wave growth, which is repeated to produce successive subpackets. Through the repetition of the subpacket formation, the wave frequency gradually increases, forming a rising-tone chorus element consisting of a series of subpackets.

An example of simulations generating chorus emission is shown in Fig. 5. This is a simulation by an electron hybrid code where cold electrons are treated as a fluid and hot energetic electrons are treated as particles undergoing cyclotron motion under a dipole magnetic field (Katoh and Omura 2006). Figure 5a shows the frequency spectra of the wave electric field and the theoretical sweep rate, in black solid line, obtained from (88) with instantaneous wave amplitude in the simulation.

\subsection{Critical distance}

Near the magnetic equator, the inhomogeneity factor $S$ is determined by the frequency sweep rate, which is nearly constant through propagation of the wave packet away from the equator. Since the dipole magnetic field is approximated by a parabolic function $\Omega_{\mathrm{e}}=\Omega_{\mathrm{e} 0}\left(1+a h^{2}\right)$ with $a=4.5 /\left(\mathrm{LR}_{\mathrm{E}}\right)^{2}$, where $\mathrm{LR}_{\mathrm{E}}$ represents the distance from the center of the Earth in the equatorial plane. The gradient of the magnetic field increases as a linear function of the distance $h$. We define the critical distance $h_{\mathrm{c}}$ at which the first term and the second term of $S$ given by (37) become equal (Omura et al. 2009). Equating the two terms and using (88), we obtain

$$
h_{\mathrm{c}}=\frac{s_{0} \omega \Omega_{\mathrm{w} 0}}{5 c a s_{2} \Omega_{\mathrm{e} 0}} \text {. }
$$

The black solid lines in Fig. 5b indicate the critical distances in the simulation by Katoh and Omura (2011). The critical distance varies as a function of the wave amplitude $\Omega_{\mathrm{w} 0}$ at the generation region near the equator. Inside the critical distance, triggering of nonlinear wave growth due to frequency variation is possible, and the region within the critical distance can be regarded as the generation region of subpackets forming chorus emissions. The critical distance is used in identifying the dominant 
term of the inhomogeneity factor $S$ as discussed in the following subsection.

\subsection{Convective wave growth}

As a chorus sub-packet propagates away from the equator, it undergoes convective wave growth due to formation of an electron hole. At a distance much greater than $h_{\mathrm{c}}$ in the downstream, we can neglect the first term on the righthand side of equation (37). Assuming the optimum wave growth condition $S=-0.4$, we obtain

$$
\Omega_{\mathrm{w}}=\frac{c s_{2}}{0.4 s_{0} \omega} \frac{\partial \Omega_{\mathrm{e}}}{\partial h}=\frac{5 \operatorname{cas}_{2} \Omega_{\mathrm{e} 0}}{s_{0} \omega} h .
$$

The ideal condition for convective wave growth can be realized if the wave amplitude increases as a linear function of $h$. Assuming the optimum condition is maintained even at a shorter distance $h\left(h<h_{\mathrm{c}}\right)$, we find the gradient of the wave amplitude

$$
\frac{\partial \Omega_{\mathrm{w}}}{\partial h}=\frac{5 \operatorname{cas}_{2} \Omega_{\mathrm{e} 0}}{s_{0} \omega} .
$$

The gradient of the wave amplitude is a condition for the optimum convective wave growth. The convective wave growth reaches a saturation when the flux of resonant electrons decreases as the absolute value of resonance velocity $\left|V_{\mathrm{R}}\right|$ increases.

More quantitative evaluation of the wave growth in space may be made by finding a steady state solution of (93). Assuming $\partial \Omega_{\mathrm{w}} / \partial t=0$, we have

$$
\frac{\partial \Omega_{\mathrm{w}}}{\partial h}=\frac{\Gamma_{\mathrm{N}}}{V_{\mathrm{g}}} \Omega_{\mathrm{w}}
$$

where we define the convective nonlinear growth rate $\Gamma_{\mathrm{N}} / V_{\mathrm{g}}$. From (92), we have

$$
\frac{\Gamma_{\mathrm{N}}}{V_{\mathrm{g}}}=\frac{Q \omega_{\mathrm{ph}}^{2}}{2 \gamma U_{t \|}}\left(\frac{\xi}{\omega \Omega_{\mathrm{w}}}\right)^{1 / 2}\left(\frac{\chi U_{\perp h}}{\pi c}\right)^{3 / 2} \exp \left(-\frac{\gamma^{2} V_{\mathrm{R}}^{2}}{2 U_{t \|}^{2}}\right),
$$

where $\omega_{\mathrm{ph}}^{2}=N_{h} e^{2} /\left(m_{0} \epsilon_{0}\right)$ and other variables are functions of $h$. Since the group velocity decreases in the frequency rage above $0.25 \Omega_{\mathrm{e}}$, as shown in Fig. 1, waves at higher frequencies undergo larger convective growth.

\subsection{Threshold wave amplitude}

Substituting the gradient of the wave amplitude (101) to the condition for the absolute instability (94), we obtain the condition for the absolute instability, i.e., triggering of the nonlinear wave growth process as

$$
\tilde{\Omega}_{\mathrm{w} 0}>\tilde{\Omega}_{\mathrm{th}},
$$

where

$$
\tilde{\Omega}_{\mathrm{th}}=\frac{100 \pi^{3} \gamma^{4} \xi}{\tilde{\omega} \tilde{\omega}_{\mathrm{ph}}^{4}\left(\chi \tilde{U}_{\perp 0}\right)^{5}}\left(\frac{\tilde{a} s_{2} \tilde{U}_{t \|}}{Q}\right)^{2} \exp \left(\frac{\gamma^{2} \tilde{V}_{\mathrm{R}}^{2}}{\tilde{U}_{t \|}^{2}}\right)
$$

where $s_{2}$ is given by (40) with $v_{\perp} / c=\tilde{U}_{\perp 0} / \gamma$. The parameters with tilde are normalized values as used in (97). The parameter of the parabolic magnetic field is normalized as $\tilde{a}=a c^{2} / \Omega_{\mathrm{e} 0}^{2}$. The wave amplitudes and frequencies are normalized by $\Omega_{\mathrm{e} 0}$ as $\tilde{\Omega}_{\mathrm{th}}=\Omega_{\mathrm{th}} / \Omega_{\mathrm{e} 0}$ and $\tilde{\omega}=\omega / \Omega_{\mathrm{e} 0}$. The velocity and momentums are normalized by the speed of light $c$ as $\tilde{V}_{\mathrm{R}}=V_{\mathrm{R}} / c$ and $\tilde{U}_{\perp 0}=U_{\perp 0} / c$. In Fig. $6 \mathrm{~b}$, we plot the optimum wave amplitude in solid lines and the threshold amplitude in dashed lines for different values of the plasma frequency $\omega_{\mathrm{pe}} / \Omega_{\mathrm{e}}=2$ (blue), 4 (green), and 8 (red) with the same parameters of energetic electrons assumed in the linear and nonlinear growth rate calculation in Fig. 6a. The optimum wave amplitude becomes higher in the lower frequency range with higher plasma frequencies.

When a triggering wave with a constant frequency $\omega_{0}$ and with an amplitude greater than the threshold amplitude (105) is present at the equator, there occurs an electron hole forming the resonant current $J_{\mathrm{B}}(<0)$ causing an frequency increase by $\delta \omega$ given by (72). The frequency increase makes the electron hole asymmetric with a finite $S$, resulting in the resonant current $J_{\mathrm{E}}(<0)$ causing wave growth at a fixed position, i.e., an absolute instability. The wave amplitude grows locally with the increased frequency forming a new wave packet detached from the triggering wave. The amplitude reaches the optimum wave amplitude (97). The wave amplitude cannot grow much greater than the optimum value, because the nonlinear growth rate becomes smaller with a larger amplitude. The dynamics of the resonant electrons also causes saturation of the nonlinear wave growth because of entrapping of resonant electrons into the wave potential filling the electron hole. When the wave amplitude is growing locally there occurs efficient entrapping of resonant electrons because of enlargement of the trapping wave potential. The trapped electrons contribute to saturation of the wave amplitude by receiving energy from the wave. After the saturation, the wave amplitude gradually decreases, because the phase organized untrapped electrons move to an opposite phase resulting in a positive $J_{\mathrm{E}}$. The subpacket with an increased frequency $\omega_{0}+\delta \omega$ propagates to the downstream undergoing the efficient convective wave growth.

\subsection{Chorus equations}

The nonlinear growth process as an absolute instability can be described by the following set of equations obtained by normalizing the wave amplitude at the equator $\Omega_{\mathrm{w} 0}$ in (88) and the frequency $\omega$ in (93) as in (97). 


$$
\frac{\partial \tilde{\omega}}{\partial \tilde{t}}=0.4 \frac{s_{0}}{s_{1}} \tilde{\omega} \tilde{\Omega}_{\mathrm{w} 0}
$$

and

$$
\begin{aligned}
\frac{\partial \tilde{\Omega}_{\mathrm{w} 0}}{\partial \tilde{t}}= & \tilde{V}_{g}\left[\frac{Q \tilde{\omega}_{\mathrm{ph}}^{2}}{2 \gamma \tilde{U}_{t \|}}\left(\frac{\xi \tilde{\Omega}_{\mathrm{w} 0}}{\tilde{\omega}}\right)^{1 / 2}\left(\frac{\chi \tilde{U}_{\perp 0}}{\pi}\right)^{3 / 2}\right. \\
& \left.\cdot \exp \left(-\frac{\gamma^{2} \tilde{V}_{\mathrm{R}}^{2}}{2 \tilde{U}_{t \|}^{2}}\right)-\frac{5 s_{2} \tilde{a}}{s_{0} \tilde{\omega}}\right]
\end{aligned}
$$

where $s_{0}, s_{1}$, and $s_{2}$ are calculated from (38), (39), and (40) with $v_{\perp} / c=\tilde{U}_{\perp 0} / \gamma$, respectively. We call these equations as "chorus equations", and we tried a simple numerical integration of the equations, as presented in Fig. 6 of Omura et al. (2009). We find a monotonic increase of the wave amplitude and frequency, in which the frequency increases rapidly like an exponential function contrary to observed chorus emissions. As it has been reported by Santolik et al. (2014), chorus wave packets consist of many subpackets. An example of a chorus rising-tone emissions is shown in Fig. 7. A rising-tone element of Fig. 7a is expanded in time, and instantaneous amplitudes and frequencies calculated from wave forms of the perpendicular wave magnetic field are plotted in Fig. 7b, c. We simulated the variation the wave amplitude and frequency using the chorus equations, as shown in Fig. 8. We integrated the equations using the parameters used in the calculation of the linear and nonlinear growth rates in Fig. 6. When the wave amplitude reached the optimum wave amplitude, we reversed the sign of the first term of (107) which corresponds to the resonant current $J_{\mathrm{E}}$. As the wave amplitude damps to a level below $\Omega_{\mathrm{th}}+0.3\left(\Omega_{\mathrm{w} 0}-\Omega_{\mathrm{th}}\right)($ rand $)$, where (rand) is a uniform random number $(0 \sim 1)$, we reversed the sign again, and the wave starts to grow. The process of wave growth and damping is repeated until the frequency reaches $0.65 \Omega_{\mathrm{e}}$. We have introduced some randomness assuming that there exist fluctuations of the electromagnetic fields which are radiated from counter-streaming energetic electrons, which are modulated in their wave phases through interaction with foregoing waves. The result is plotted in Fig. 8. The observed wave amplitudes of subpackets in Fig. 7a are greater than those of the modeled wave amplitudes shown in Fig. 8b. This is probably because of the convective wave growth from the source to the spacecraft.

\subsection{Formation of chorus element}

In the model of nonlinear wave growth presented above, we assumed nonlinear wave growth takes place at the equator. As we find in Fig. 5b, formation of each subpacket takes place at different places around the equator. A wave packet produced by the triggering of the nonlinear wave growth is relatively short and the frequency increase is only by a small increment given by $\delta \omega$, i.e., the frequency $\omega_{1}=\omega_{0}+\delta \omega$. The wave packet propagates away from the triggering point with a wave number $k_{1}$ undergoing the convective nonlinear wave growth due to the electron hole in the velocity phase space. The wave packet with $k_{1}$ interacts with counter streaming resonant electrons going around the electron hole as untrapped resonant electrons. The amplitude of the wave packet reaches a substantially large amplitude. The counter streaming untrapped resonant electrons going though the electron hole are organized in phase with the wave number of the wave packet $k_{1}$. It is noted that the frequency of a wave packet moving with the group velocity does not change in the absence of the resonant current as indicated by (10). The group of electrons in resonance with the wave packet are strongly modulated in gyro-phase with a wave number $k_{1}$. The phase-modulated electrons move to the upstream region keeping the information of the new wave number $k_{1}$. These electrons can work as an antenna which can radiate a helical wave with a new frequency $\omega_{1}$ that satisfies the local dispersion relation with the wave number $k_{1}$. The helical wave works as a new triggering wave for the next cycle of the nonlinear wave growth. The triggering process is repeated sequentially with slightly different frequencies. A model of the subpacket formation has been proposed based on the chorus equations integrated repeatedly at slightly different positions moving to the upstream region gradually. The model has reproduced the observed feature that the wave frequency drops between subpackets (Hanzelka et al. 2020). The tendency for points of the subpacket formation to shift to the upstream from the equator is often found in the simulation suggesting the sequential triggering as suggested by the model, but this is not always the case in the particle simulations, as presented in Fig. 3 of Hikishima et al. (2009).

In each process of the nonlinear wave growth, the wave amplitude saturates around the optimum wave amplitude. Therefore the spectrum of the chorus emission near the equator follows the profile of the optimum wave amplitude as a function of frequency. The optimum amplitude decreases at higher frequencies. When the optimum amplitude becomes less than the threshold amplitude, the nonlinear wave growth cannot take place. The frequency range of chorus emissions is determined from the relation of the optimum and threshold amplitudes. Since the threshold amplitude also decreases at higher frequency in most cases, the highest frequency of chorus elements is determined by another mechanism such as the cyclotron damping near the electron 

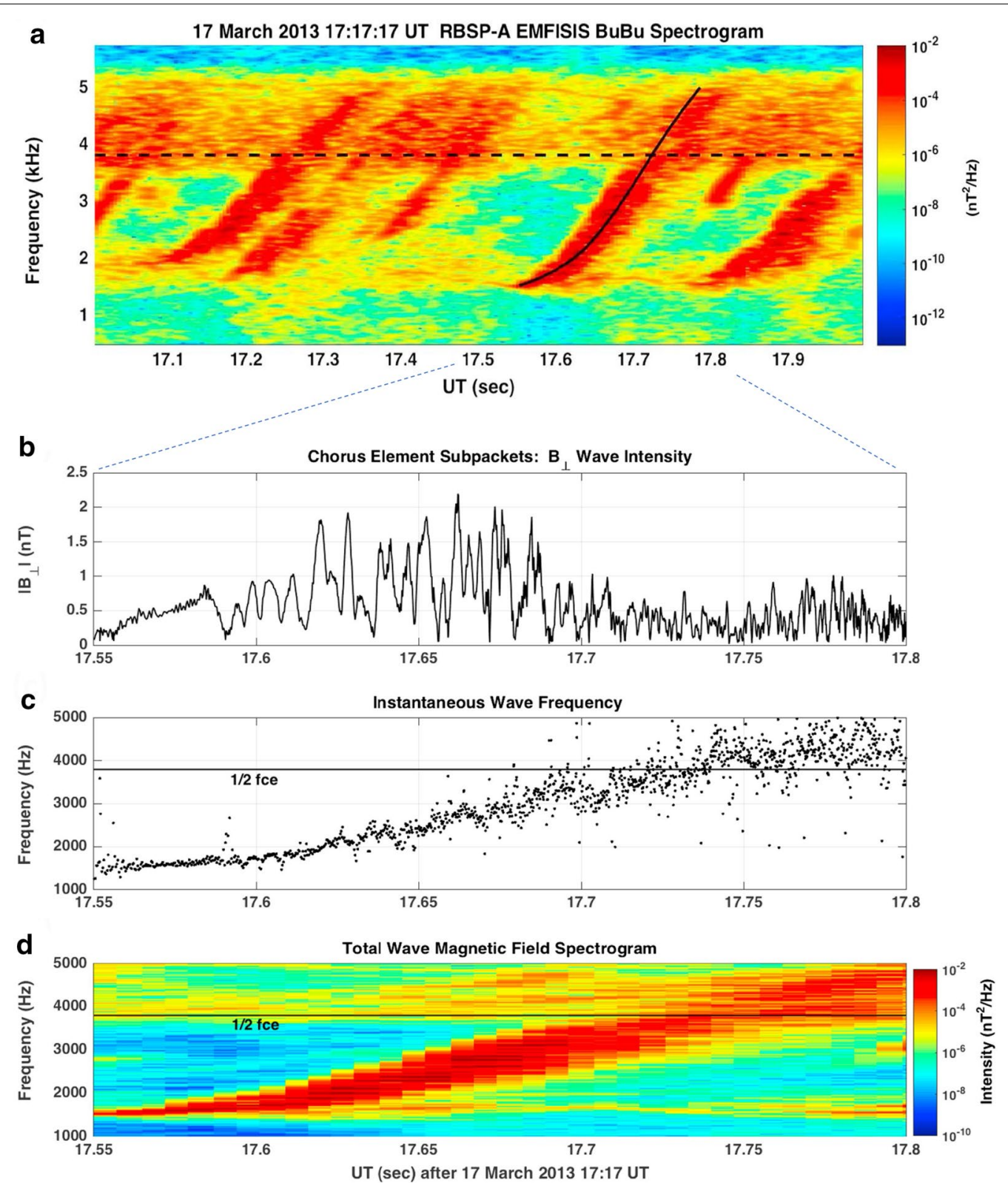

Fig. 7 Chorus observation. Chorus emissions and sub-packet structures of a single element observed by Van Allen Probe A (after Foster et al. (2017))

cyclotron frequency. The formation process suggested above is confirmed by an observation of chorus emissions by THEMIS spacecraft (Kurita et al. 2012). The process is also confirmed by simulation studies, as shown in Fig. 6 of Katoh and Omura (2013), and Fig. 3 of Katoh and Omura (2016).

\subsection{Plasmaspheric hiss}

The threshold amplitude $\Omega_{\text {th }}$ for the nonlinear wave growth strongly depends on the gradient of the magnetic field as we find $a^{2}$ in (105). Katoh and Omura (2013) studied the effect of the gradient of the magnetic field on generation process of chorus and broadband hiss-like 


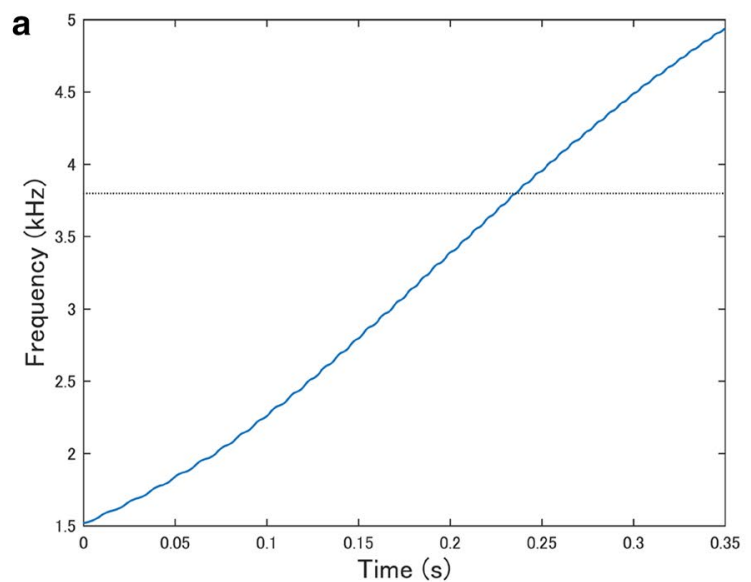

b

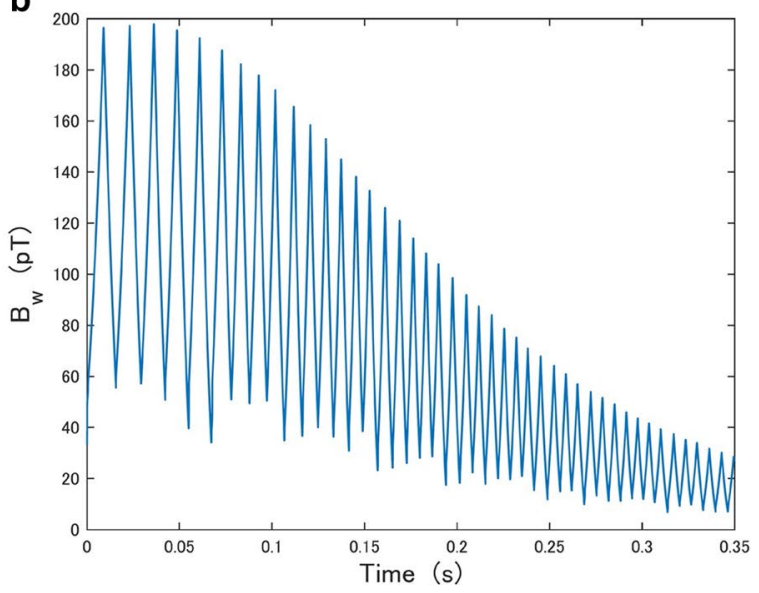

C

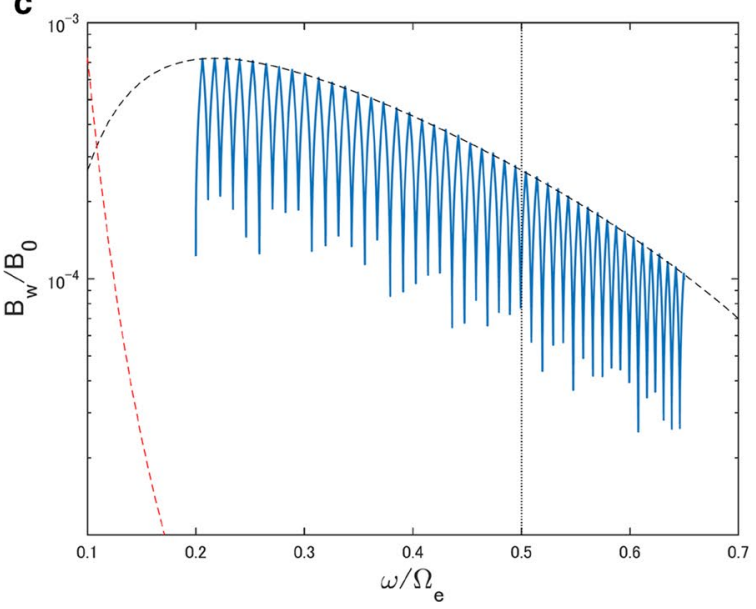

Fig. 8 Chorus model. Formation of sub-packets of a single chorus emission at the equator with $V_{\perp 0}=0.45 \mathrm{c}$ and $U_{t \|}=0.25 \mathrm{c}$

emissions. For the small gradient case, the threshold amplitude becomes very low, and there arises a big gap between the optimum and threshold amplitudes allowing the nonlinear wave growth process occurs in wide range of the amplitudes and frequencies. In the simulations by the electron hybrid code (Katoh and Omura 2007), we find broadband hiss-like emissions for the small gradient case, in which we find many rising-tone emissions and some falling-tone emissions with shorter duration periods being generated. With larger gradient, the threshold amplitude becomes greater than the optimum amplitude, and generation of rising-tone emissions are suppressed.

The generation of these emissions with frequency variation is due to a coherent wave that modify the velocity distribution function $F\left(v_{\|}\right)$with its wave potential formed at the cyclotron resonance velocity $V_{\mathrm{R}}$ as we studied in the previous sections. Depending on the numbers of trapped and untrapped resonant electrons, we have either an electron hole or an electron hill as shown in Fig. 9a. When we have depletion of trapped electrons, an electron hole is generated, and we find more resonant electrons in the direction of the wave magnetic field vector $\boldsymbol{B}_{\mathrm{w}}$, which gives rise to a negative $J_{\mathrm{B}}$ inducing the frequency increase as indicated by (74). As the frequency increases, the absolute value of the resonance velocity decreases shifting to the higher density part of the velocity distribution function making the hole deeper. Because of the rising-tone frequency, the shape of the electron hole is distorted, as shown in Fig. 9b, which make the perpendicular velocities of the untrapped resonant electrons gathered in the direction of the wave electric field vector $\boldsymbol{E}_{\mathrm{w}}$, resulting in a negative $J_{\mathrm{E}}$ for the wave growth. On the other hand, when we have enhancement of trapped electrons, an electron hill is formed, and we find more resonant electrons in the opposite direction of $\boldsymbol{B}_{\mathrm{w}}$ giving rise to a positive $J_{\mathrm{B}}$ inducing the frequency decrease. As the frequency decreases, the absolute value of the resonance velocity increases shifting to the lower density part of the distribution function. The electron hill formed by the trapped resonant electrons is more enhanced with a less number of untrapped resonant electrons outside the trapping wave potential. Because of the distortion of the trapping potential due to the frequency decrease, the perpendicular velocities of trapped electrons are in the direction of $\boldsymbol{E}_{\mathrm{w}}$ forming a negative $J_{\mathrm{E}}$ for the wave growth. Therefore, the velocity distribution function $F\left(v_{\|}\right)$ is unstable in the presence of the coherent wave both for rising-tone and falling-tone triggered waves. As we have studied the convective wave growth, rising-tone emissions have a better chance of the wave growth because of the increasing gradient of the magnetic field in the downstream from the equator.

In the plasmasphere, the ratio of the electron plasma frequency $\omega_{\mathrm{pe}}$ to the electron cyclotron frequency $\Omega_{\mathrm{e}}$ is much increased to $15-25$, while the ratio is $2-5$ outside the plasmasphere. The ratio controls the frequency range over which the nonlinear wave growth takes place, as shown 

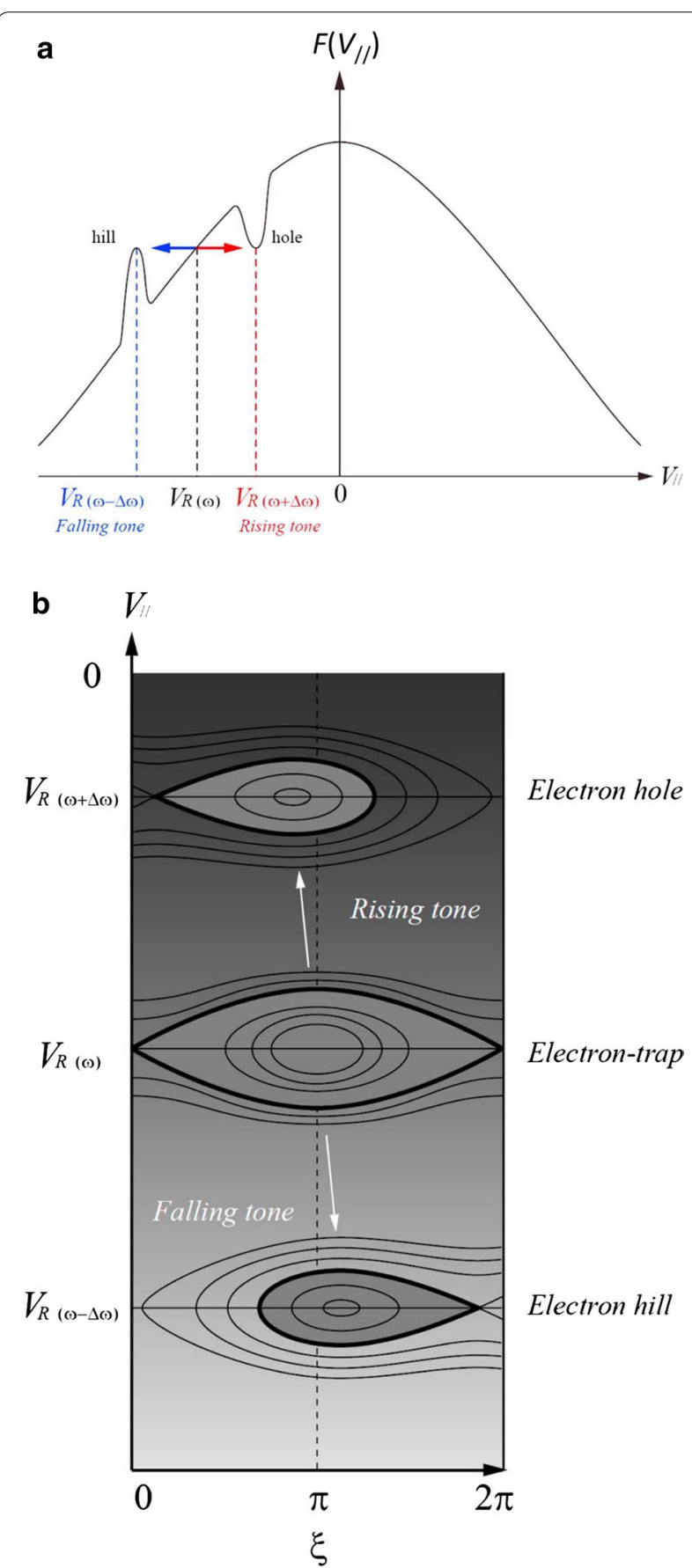

Fig. 9 Triggering of rising and falling tone emissions. Velocity distribution function unstable to a coherent triggering wave. a Variation of resonance velocities of rising-tone and falling tone emissions. $\mathbf{b}$ Electron hole and hill in the velocity phase space giving growth to rising-tone and falling-tone emissions (after Omura et al. (2015a)) in Fig. 10a. The frequency range over which the optimum amplitude (solid line) is greater than the threshold amplitude (dashed line) shifts to the lower frequency range as the plasma frequency increases $\left(\omega_{\mathrm{pe}} / \Omega_{\mathrm{e}}=5,15,25\right)$. We plot frequencies in $\mathrm{Hz}$ assuming the electron cyclotron frequency $f_{\mathrm{ce}}=9 \mathrm{kHz}$. The large $\omega_{\mathrm{pe}} / \Omega_{\mathrm{e}}$ makes the frequency ranges of the nonlinear wave growth much lower $\left(<0.1 \Omega_{\mathrm{e}}\right)$. As we have seen in the case of hiss-like emissions, we can have formation of many sub-packets at the same time and the same position, when the threshold amplitude is much smaller than the optimum wave amplitude. The nonlinear transition time for formation of the resonant currents, which is calculated from the optimum wave amplitude, becomes shorter as the plasma frequency increases, as shown in Fig. 10b. This suggests the time scales of the generation of hiss waves are shorter than those of chorus emissions.

Initially we may need a seed wave that may grow from the linear instability or external sources such as whistlers or chorus coming from the outside of the plasmasphere. Once the nonlinear wave growth is initiated by a triggering wave, triggering of short wave packet expands over the entire frequency range over which the optimum amplitude is greater than the threshold amplitude. Because of the concurrent triggering, the wave frequency spectra look like noisy incoherent waves. The frequencies of these sub-packets are usually well separated each other so that their wave potentials do not overlap in the velocity phase space. Using the cyclotron resonance condition (21), we can calculate the minimum frequency separation $\Delta \omega$ corresponding to twice of the trapping velocity $V_{\operatorname{tr}}$ as

$$
\Delta \omega=\left(\frac{\mathrm{d} V_{\mathrm{R}}}{\mathrm{d} \omega}\right)^{-1}\left(2 V_{\mathrm{tr}}\right)
$$

From the resonance condition (21), we have

$$
\frac{\mathrm{d} V_{\mathrm{R}}}{\mathrm{d} \omega}=\frac{1}{k}+\frac{1}{k^{2}}\left(\frac{\Omega_{\mathrm{e}}}{\gamma}-\omega\right) V_{\mathrm{g}}^{-1} .
$$

Using $V_{\mathrm{tr}}=2 \omega_{\mathrm{tr}} / k$ and (109), we obtain

$$
\Delta \omega=4 \omega_{\operatorname{tr}}\left\{1+\chi^{2}\left(\frac{\Omega_{\mathrm{e}}}{\gamma \omega}-1\right)\left[\xi^{2}+\frac{\Omega_{\mathrm{e}}}{2\left(\Omega_{\mathrm{e}}-\omega\right)}\right]\right\}^{-1} .
$$

When the frequencies of two wave packets adjacent in frequency are separated much greater than $\Delta \omega$, which we call the separability condition, the resonant interaction of each of the waves with energetic electrons is not affected by other waves. The interaction is the same as in 


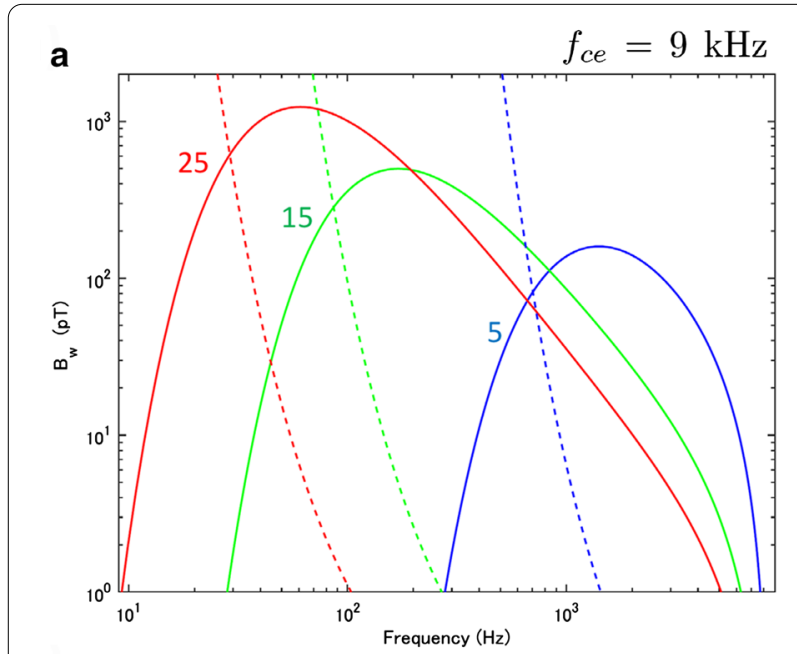

the case of a single wave interacting with energetic electrons. Using the optimum wave amplitude, we calculated $\Delta \omega$ for different $\omega_{\text {pe }} / \Omega_{\mathrm{e}}$, as plotted in Fig. 10c. The bandwidth in Fig. 3b of Omura et al. (2015a) was calculated with the threshold wave amplitude, showing that the separability condition is well satisfied at the moment of triggering of the nonlinear wave growth.

\subsection{Particle simulation of hiss emissions}

A particle simulation has been conducted by using a particle code that was studied for chorus simulations (Hikishima et al. 2009) with the plasma frequency $\omega_{\mathrm{pe}}=15 \Omega_{\mathrm{e} 0}$ (Hikishima et al. 2020). The simulation reproduced the generation process of hiss emissions, as shown in Fig. 11. The simulation was started with electromagnetic thermal noise due to limited number of super particles representing the dense cold elec-

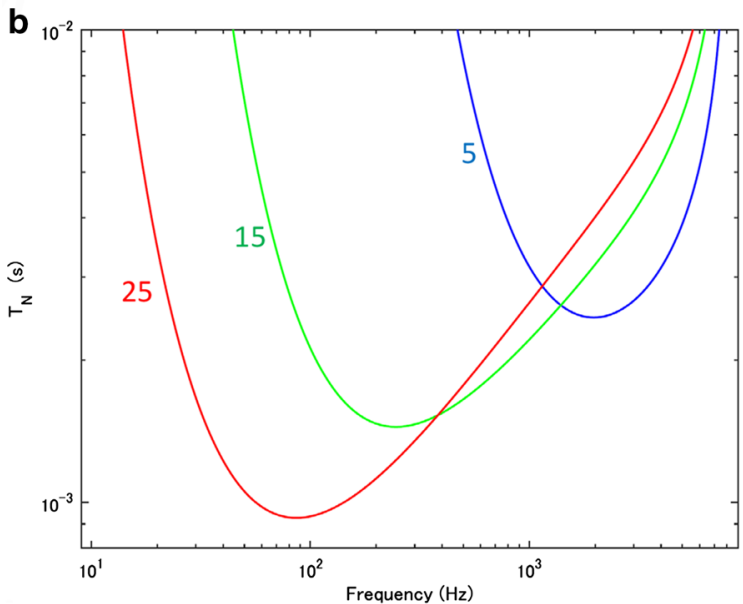
trons and energetic hot electrons with the density ratio

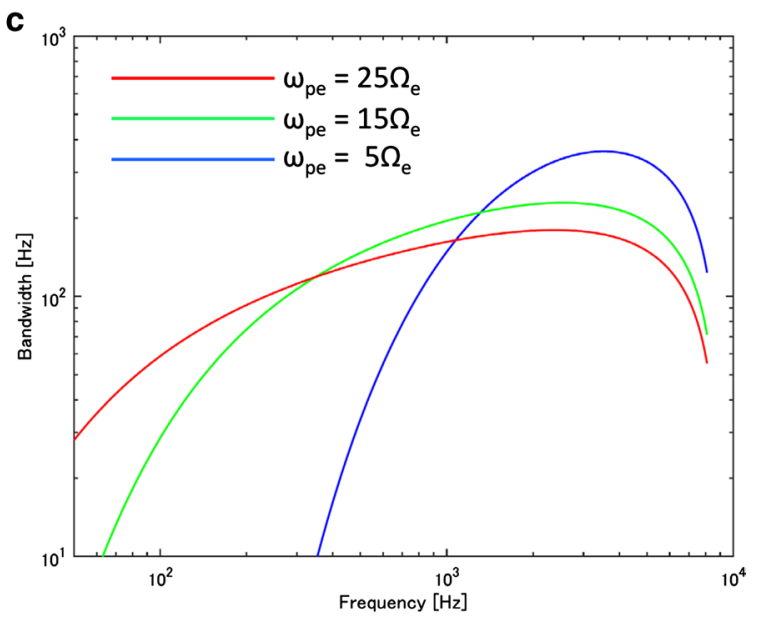

Fig. 10 Properties of hiss emissions based on nonlinear wave growth theory. a Optimum (solid lines) and threshold (dashed lines) amplitudes, $\mathbf{b}$ nonlinear transition time $T_{N}$ with $\tau=0.5$, and c bandwidth as coherent waves for different cold plasma densities specified by the electron plasma frequency $f_{\text {pe }}$ normalized by the cyclotron frequency $f_{\text {ce }}=9 \mathrm{kH}$

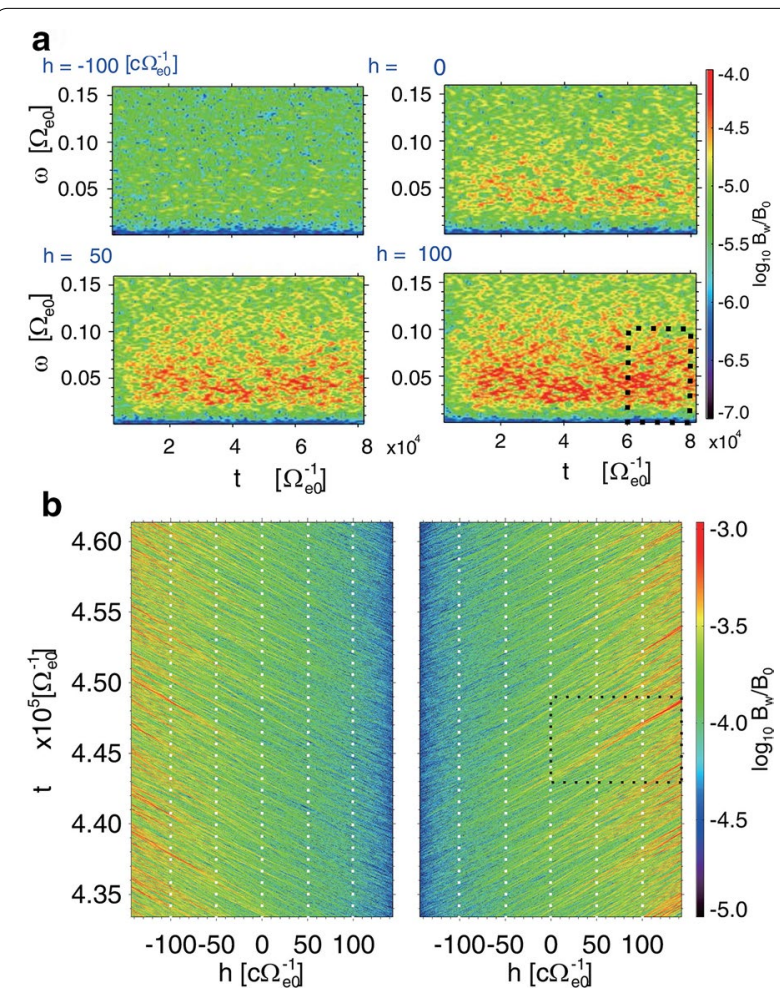

Fig. 11 Particle simulation of hiss emissions. a Dynamics frequency spectra of waves propagating forward in the direction of the magnetic field at positions $h=-100,0,50,100 \mathrm{c} \Omega_{\mathrm{e} 0}^{-1}$ at the initial phase of the simulation $\left(t=0 \sim 8 \times 10^{4} \Omega_{e 0}^{-1}\right)$. The equator is at $h=0$. $\mathbf{b}$ Spatial and temporal evolution of transverse wave amplitudes $B_{w}$ of waves propagating forward (right panel) and backward (left panel) along the magnetic field line for an short time interval $t=4.35 \sim 4.60 \times 10^{5} \Omega_{\mathrm{e} 0}^{-1}$ (after Hikishima et al. (2020)) 
$n_{\mathrm{h}} / n_{\mathrm{c}}=4 \times 10^{-4}$. Hot electrons have a temperature anisotropy given by thermal momentums $U_{t \|}=0.25 \mathrm{c}$ and $U_{t \perp}=0.4 c$ with $\beta=0.3$ for the subtracted-Maxwellian distribution at the equator. The transverse waves in the simulations are separated into forward and backward waves based on their spatial helicity as whistler-mode waves. Figure 11a shows the initial phase of the generation process where waves are gradually excited because of the positive linear growth rate and subsequent nonlinear wave growth process with frequency variations. Small scale structures with rising-tone and falling-tone frequencies grow concurrently at different frequencies. At a position $h=100 c \Omega_{\mathrm{e} 0}^{-1}$ away from the equator, the nonlinear convective growth makes the wave packet significantly larger than those near the equator. The generation of the wave packets, which we call hiss elements, continues for a long time, as shown in Fig. 11b. The spatial and temporal profile of hiss elements during a relatively short period of $4.35 \sim 4.60 \times 10^{5} \Omega_{\mathrm{e} 0}^{-1}$. We find many discrete hiss elements propagating with different group velocities corresponding to different wave frequencies. We can understand that hiss emission consists of many discrete wave packets (hiss elements), which of them are undergoing the nonlinear wave-particle interaction as we find in the generation process of chorus emissions.

\subsection{Coherency and incoherency}

Coherency is defined in different ways in different cases. In the case of a wave particle interaction, the interaction is called coherent when particles interact with a wave having a smooth variation of the amplitude and the wave phase. Even with two waves whose resonance velocities are well separated in the velocity phase space as we assumed in the separability condition of hiss elements, the interaction is coherent when the particles interact with one of the waves at a time. Particles interacting with a single wave undergo nonlinear trapping motion when the parallel velocity of a particle is within the range of trapping velocity from the resonance velocity

$$
V_{\mathrm{R}}-V_{\mathrm{tr}}<v_{\|}<V_{\mathrm{R}}+V_{\mathrm{tr}} .
$$

The velocity range shown above is the width of the trapping wave potential in the velocity phase space, which we call the trapping region. When $\left|v_{\|}-V_{\mathrm{R}}\right| \gg V_{\mathrm{tr}}$, a particle hardly feels the effect of the wave, undergoing an adiabatic motion with very small perturbation. When resonance velocities of the two waves are close to each other, the trapping regions of the waves overlap. The particle motion becomes chaotic when it is under the direct influences of two waves with different frequencies. The particle motion becomes incoherent with the wave structures. In this case, we describe the wave-particle interaction as incoherent, when there occurs overlapping of trapping potentials in the velocity phase space. The trapping velocity $V_{\mathrm{tr}}$ of a whistler mode waves in the parallel propagation depends on both wave amplitude $B_{\mathrm{w}}$ and perpendicular velocity of a resonant electron $v_{\perp}$, because the trapping potential is formed by the Lorentz force $-e \boldsymbol{v}_{\perp} \times \boldsymbol{B}_{\mathrm{w}}$. Therefore, the coherency of the cyclotron wave-particle interaction also depends on the particle property $v_{\perp}$. In the quasi-linear diffusion theory, many waves forming a band of wave spectra are assumed, and the waves are incoherent for resonant electrons because of overlapping of trapping potentials of the waves.

\section{Oblique whistler mode wave-particle interaction 4.1 Dispersion relation and group velocity of oblique whistler-mode wave}

As the wave packet propagates away from the equator, the wave normal angle becomes gradually oblique because of the curvature of the magnetic field. The cold plasma dispersion relation of an oblique whistler mode wave is given from the Appleton-Hartree equation (e.g., Helliwell (1993)) as

$$
\begin{aligned}
& D\left(\omega, k_{z}, k_{x}\right)=\frac{c^{2}\left(k_{z}^{2}+k_{x}^{2}\right)}{\omega^{2}}-1 \\
& \quad+\frac{2 X(1-X)}{2(1-X)-Y^{2} \sin ^{2} \theta \pm Y \sqrt{Y^{2} \sin ^{4} \theta+4(1-X)^{2} \cos ^{2} \theta}}=0,
\end{aligned}
$$

where $X=\omega_{\mathrm{pe}}^{2} / \omega^{2}, Y=\Omega_{\mathrm{e}} / \omega$, and $\theta$ is the angle between the background magnetic field and the wave vector. We take the direction of the ambient magnetic field in the $z$-direction, keeping the wave number vectors in the plane formed by $x$ and $z$ axes, as shown in Fig. 12a. We assume that the plasma density and the magnetic field are constant in time, namely, $\partial X / \partial t=0$, and $\partial Y / \partial t=0$. In the absence of nonlinear resonant currents, we assume $D\left(\omega, k_{z}, k_{x}\right)=0$ is always satisfied for all combinations of $\omega, k_{z}$, and $k_{x}$. In observing chorus emissions at a fixed position in space, we find $\partial \omega / \partial t \neq 0$, then we have $\partial k_{z} / \partial t \neq 0$ and $\partial k_{z} / \partial t \neq 0$ to make propagation of the wave possible with $D=0$. Therefore, we have 


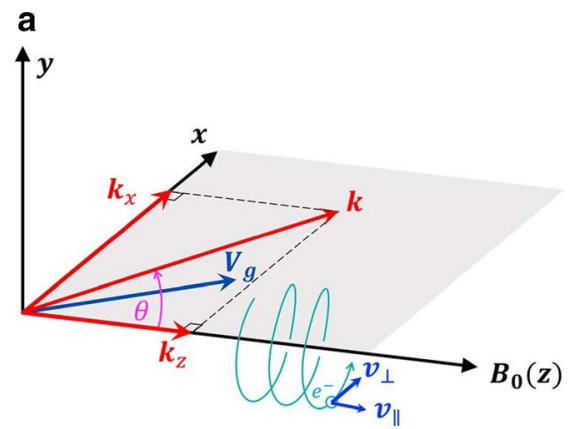

b

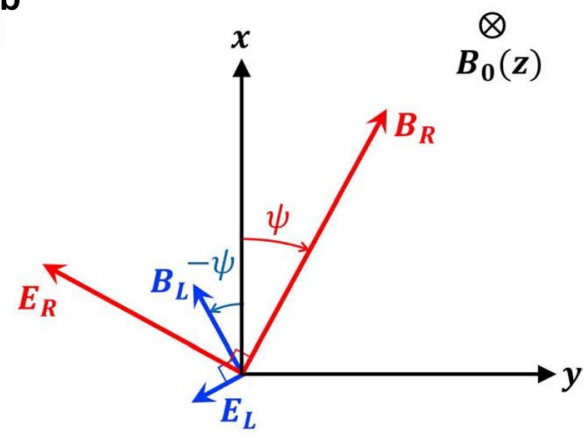

Fig. 12 Vector relations of oblique whistler-mode wave. a Configuration of vectors of the oblique whistler mode waves interacting with a resonant electron. $\mathbf{b}$ Wave vectors of right-hand circularly polarized wave (in red) and left-hand circularly polarized wave (in blue) in a plane perpendicular to the background magnetic field (after Omura et al. (2019))

$$
\frac{\partial D}{\partial t}=\frac{\partial D}{\partial \omega} \frac{\partial \omega}{\partial t}+\frac{\partial D}{\partial k_{z}} \frac{\partial k_{z}}{\partial t}+\frac{\partial D}{\partial k_{x}} \frac{\partial k_{x}}{\partial t}=0 .
$$

Noting that $\omega, k_{z}$, and $k_{x}$ are defined as derivatives of the wave phase $\psi$ as $\omega=\partial \psi / \partial t, k_{z}=-\partial \psi / \partial z$, and $k_{x}=-\partial \psi / \partial x$, respectively, we find by changing the order of differential operations

$$
\frac{\partial k_{z}}{\partial t}=-\frac{\partial \omega}{\partial z}, \frac{\partial k_{x}}{\partial t}=-\frac{\partial \omega}{\partial x} .
$$

Multiplying (113) by $\partial \omega / \partial D$, and substituting (114), we obtain

$$
\frac{\partial \omega}{\partial t}-\left(\frac{\partial D}{\partial k_{z}} \frac{\partial \omega}{\partial D}\right) \frac{\partial \omega}{\partial z}-\left(\frac{\partial D}{\partial k_{x}} \frac{\partial \omega}{\partial D}\right) \frac{\partial \omega}{\partial x}=0 .
$$

We define the parallel and perpendicular components of the group velocity $V_{\mathrm{g}}$ as $V_{\mathrm{g} \|}$ and $V_{\mathrm{g} \perp \text {. Since the frequency }}$ is constant in the frame of reference moving with the group velocity, we have

$$
\frac{\partial \omega}{\partial t}+V_{\mathrm{g} \|} \frac{\partial \omega}{\partial z}+V_{\mathrm{g} \perp} \frac{\partial \omega}{\partial x}=0 .
$$

Comparing (115) and (116), we obtain

$$
V_{\mathrm{g} \|}=-\frac{\partial D}{\partial k_{z}}\left(\frac{\partial D}{\partial \omega}\right)^{-1}, V_{\mathrm{g} \perp}=-\frac{\partial D}{\partial k_{x}}\left(\frac{\partial D}{\partial \omega}\right)^{-1} .
$$

The lengthy derivatives of $D$ in (117) are presented in Appendix A of Hsieh and Omura (2017), where " $Y Y_{\omega}$ " in Equation A21 needs to be replaced by " $2 Y Y_{\omega}$ ". Using (117), we can calculate $\theta_{\mathrm{gB}}$, angle of group velocity $\boldsymbol{V}_{\mathrm{g}}$ with respect to the background magnetic field, as a function of frequency $\omega$ for different wave normal angles $\theta$, as plotted in Fig. 13a.

\subsection{Decomposition of oblique wave}

We can write down an oblique whistler-mode wave by the following vector relations (Bell 1984; Nunn and Omura 2015; Omura et al. 2019). The wave electric field is expressed by

$$
\boldsymbol{E}_{\mathrm{w}}=\boldsymbol{e}_{x} E_{x}^{\mathrm{w}} \sin \psi-\boldsymbol{e}_{y} E_{y}^{\mathrm{w}} \cos \psi+\boldsymbol{e}_{z} E_{z}^{\mathrm{w}} \sin \psi
$$

and the wave magnetic field is given by

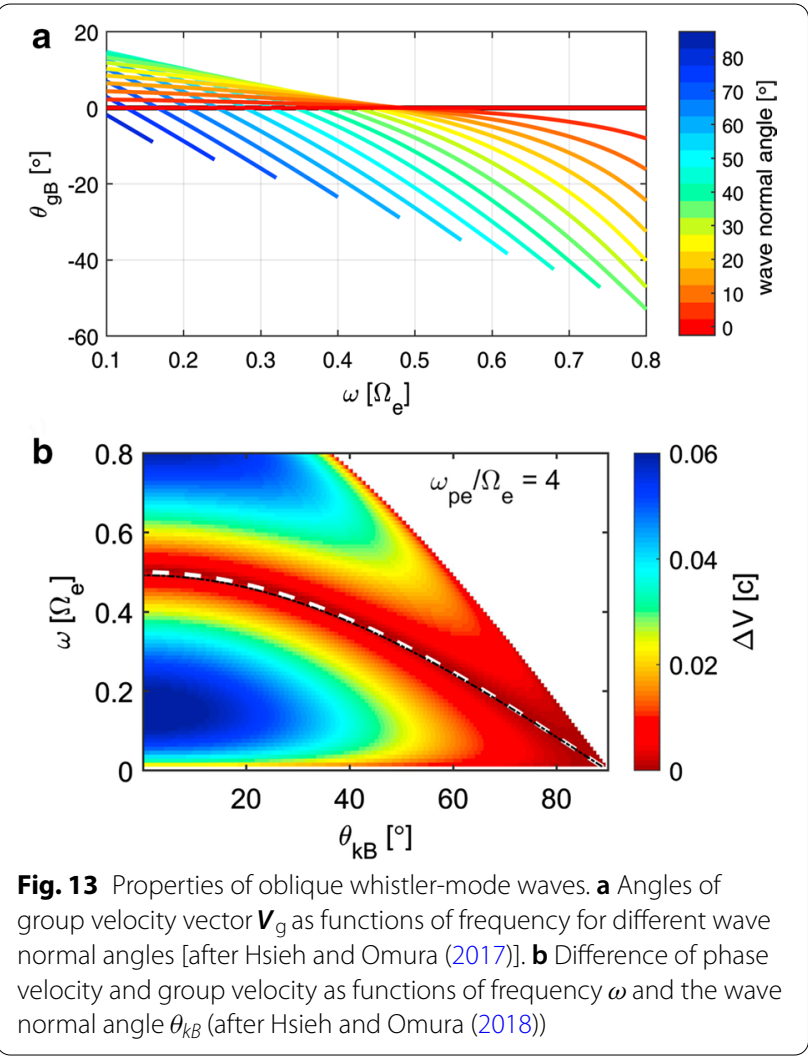




$$
\boldsymbol{B}_{\mathrm{w}}=\boldsymbol{e}_{x} B_{x}^{\mathrm{w}} \cos \psi+\boldsymbol{e}_{y} B_{y}^{\mathrm{w}} \sin \psi-\boldsymbol{e}_{z} B_{z}^{\mathrm{w}} \cos \psi
$$

where $\boldsymbol{e}_{x}, \boldsymbol{e}_{y}$, and $\boldsymbol{e}_{z}$ are unit vectors in the directions of $x$, $y$, and $z$, axes, respectively. We assume $E_{x}^{\mathrm{w}}, E_{y}^{\mathrm{w}}, E_{z}^{\mathrm{w}}, B_{x}^{\mathrm{w}}$, $B_{y}^{\mathrm{w}}$, and $B_{z}^{\mathrm{w}}$ are positive amplitudes of the electromagnetic wave fields in the $x, y$, and $z$ directions, which satisfy the cold plasma dispersion relation. These amplitudes are expressed as functions of the wave magnetic field amplitude $B_{\mathrm{w}}$ as derived in the Appendix B of Hsieh and Omura (2017). The wave phase $\psi$ is given by

$$
\psi=\omega t-k_{x} x-k_{z} z+\text { const., }
$$

where $\omega, k_{x}$, and $k_{z}$ are the angular frequency, perpendicular wave number, and parallel wave number of the wave, respectively. The signs of the components of the wave field are determined based on Maxwell's equations:

$$
\boldsymbol{k} \times \boldsymbol{E}_{\mathrm{w}}=\omega \boldsymbol{B}_{\mathrm{w}},
$$

where $\boldsymbol{k}=\left(k_{x}, 0, k_{z}\right)$. From (121), we obtain the relations among each component of the wave field as

$$
\begin{aligned}
& B_{x}^{\mathrm{w}}=\frac{k_{z}}{\omega} E_{y}^{\mathrm{w}}, \\
& B_{y}^{\mathrm{w}}=\frac{1}{\omega}\left(k_{z} E_{x}^{\mathrm{w}}-k_{x} E_{z}^{\mathrm{w}}\right), \\
& B_{z}^{\mathrm{w}}=\frac{k_{x}}{\omega} E_{y}^{\mathrm{w}} .
\end{aligned}
$$

These relations satisfy the equation:

$$
\boldsymbol{E}_{\mathrm{W}} \cdot \boldsymbol{B}_{\mathrm{w}}=0
$$

We calculate the Poynting flux $\boldsymbol{P}=\boldsymbol{E}_{\mathrm{W}} \times \boldsymbol{B}_{\mathrm{W}} / \mu_{0}$ to find the flow direction of the wave energy. Each component of $\boldsymbol{P}=\left(P_{x}, P_{y}, P_{z}\right)$ is given by

$$
\begin{aligned}
& P_{x}=\left[E_{y}^{\mathrm{w}} B_{z}^{\mathrm{w}} \cos ^{2} \psi-E_{z}^{\mathrm{w}} B_{y}^{\mathrm{w}} \sin ^{2} \psi\right] / \mu_{0}, \\
& P_{y}=\left[\left(E_{z}^{\mathrm{w}} B_{x}^{\mathrm{w}}+E_{x}^{\mathrm{w}} B_{z}^{\mathrm{w}}\right) \sin \psi \cos \psi\right] / \mu_{0}, \\
& P_{z}=\left[E_{x}^{\mathrm{w}} B_{y}^{\mathrm{w}} \sin ^{2} \psi+E_{y}^{\mathrm{w}} B_{z}^{\mathrm{w}} \cos ^{2} \psi\right] / \mu_{0} .
\end{aligned}
$$

Since $P_{z}>0$, the Poynting vector is directed toward the positive direction of the background magnetic field. The sign of $P_{x}$ changes depending on the wave normal angle $\theta$ and the wave phase $\psi$. Averaging over the one cycle of the wave phase, $P_{x}$ takes a finite value that depends on $\theta$, while $P_{y}$ becomes zero on average over $\psi$. This is consistent with the fact that the group velocity remains on the plane formed by the wave magnetic field and the wave normal vector.

The perpendicular fields of the electromagnetic wave at an oblique angle represented by $\boldsymbol{E}_{\mathrm{w} \perp}$ and $\boldsymbol{B}_{\mathrm{w} \perp}$ defined above are decomposed into two circularly polarized waves as shown in Fig. 12b. The amplitudes of the two circularly polarized waves are given by

$$
\begin{aligned}
& E_{\mathrm{R}}^{\mathrm{w}}=\frac{E_{x}^{\mathrm{w}}+E_{y}^{\mathrm{w}}}{2}, E_{\mathrm{L}}^{\mathrm{w}}=\frac{E_{y}^{\mathrm{w}}-E_{x}^{\mathrm{w}}}{2}, \\
& B_{\mathrm{R}}^{\mathrm{w}}=\frac{B_{x}^{\mathrm{w}}+B_{y}^{\mathrm{w}}}{2}, B_{\mathrm{L}}^{\mathrm{w}}=\frac{B_{x}^{\mathrm{w}}-B_{y}^{\mathrm{w}}}{2} .
\end{aligned}
$$

The field vectors of the R-mode wave are given by

$$
\begin{aligned}
& \boldsymbol{E}_{\mathrm{R}}=E_{\mathrm{R}}^{\mathrm{W}}\left[\boldsymbol{e}_{x} \sin \psi-\boldsymbol{e}_{y} \cos \psi\right], \\
& \boldsymbol{B}_{\mathrm{R}}=B_{\mathrm{R}}^{\mathrm{w}}\left[\boldsymbol{e}_{x} \cos \psi+\boldsymbol{e}_{y} \sin \psi\right],
\end{aligned}
$$

while the vectors of the L-mode are given by

$$
\begin{aligned}
& \boldsymbol{E}_{\mathrm{L}}=E_{\mathrm{L}}^{\mathrm{w}}\left[\boldsymbol{e}_{x} \sin (-\psi)-\boldsymbol{e}_{y} \cos (-\psi)\right], \\
& \boldsymbol{B}_{\mathrm{L}}=B_{\mathrm{L}}^{\mathrm{w}}\left[\boldsymbol{e}_{x} \cos (-\psi)+\boldsymbol{e}_{y} \sin (-\psi)\right] .
\end{aligned}
$$

Both $\left(\boldsymbol{E}_{\mathrm{R}}, \boldsymbol{B}_{\mathrm{R}}\right)$ and $\left(\boldsymbol{E}_{\mathrm{L}}, \boldsymbol{B}_{\mathrm{L}}\right)$ satisfy (121) and (125). From the variation of the wave phase $\psi$, we can understand $\boldsymbol{E}_{\mathrm{R}}$ and $\boldsymbol{B}_{\mathrm{R}}$ are a circularly polarized wave rotating in the same direction of electron cyclotron motion, and they can be in resonance with electrons with the effect of Doppler shift and the relativistic effect that makes the frequency of the cyclotron motion smaller.

To express all electromagnetic field components in terms of the wave magnetic field, we define the ratios of the wave electric field to the wave magnetic field as

$$
U_{\mathrm{R}}=E_{\mathrm{R}}^{\mathrm{w}} / B_{\mathrm{R}}^{\mathrm{w}}, \quad U_{\mathrm{L}}=E_{\mathrm{L}}^{\mathrm{w}} / B_{\mathrm{L}}^{\mathrm{w}} .
$$

Using (121) and (135), we solve for the parallel component $E_{z}^{\mathrm{w}}$ and $B_{z}^{\mathrm{w}}$, and we obtain

$$
\begin{aligned}
& E_{z}^{\mathrm{w}}=\frac{k_{z}}{k_{x}}\left[\left(U_{\mathrm{R}}-V_{\mathrm{p} \|}\right) B_{\mathrm{R}}^{\mathrm{w}}-\left(U_{\mathrm{L}}-V_{\mathrm{p} \|}\right) B_{\mathrm{L}}^{\mathrm{w}}\right], \\
& B_{z}^{\mathrm{w}}=\frac{k_{x}}{\omega}\left(U_{\mathrm{R}} B_{\mathrm{R}}^{\mathrm{w}}+U_{\mathrm{L}} B_{\mathrm{L}}^{\mathrm{w}}\right),
\end{aligned}
$$

where $V_{\mathrm{p} \|}=\omega / k_{z}$.

An oblique whistler-mode wave is decomposed into three electromagnetic waves, i.e., a circularly polarized R-mode wave $\left(E_{\mathrm{R}} \mathrm{e}^{i(\psi-\pi / 2)}, B_{\mathrm{R}} \mathrm{e}^{i \psi}\right)$, and a circularly 
polarized L-mode wave $\left(E_{\mathrm{L}} \mathrm{e}^{i(-\psi-\pi / 2)}, B_{\mathrm{L}} \mathrm{e}^{-i \psi}\right)$, and another wave $\left(E_{z}^{\mathrm{w}} \sin \psi,-B_{z}^{\mathrm{w}} \cos \psi\right)$ in the parallel direction.

\subsection{Oblique wave-particle interaction}

Substituting these waves into the right hand sides of the equations of motions (15) and (16), we obtain

$$
\sin \zeta_{\mathrm{L}}=-\sum_{n=-\infty}^{\infty} J_{n+1}(\beta) \sin \left(n \phi-\psi_{B}\right)
$$

$$
\sin \psi=-\sum_{n=-\infty}^{\infty} J_{n}(\beta) \sin \left(n \phi-\psi_{B}\right)
$$

$$
\begin{aligned}
& \frac{\mathrm{d}\left(\gamma v_{\|}\right)}{\mathrm{d} t}=\frac{e}{m_{0}}\left[v_{\perp} B_{\mathrm{R}}^{\mathrm{w}} \sin \zeta_{\mathrm{R}}+v_{\perp} B_{L}^{\mathrm{w}} \sin \zeta_{\mathrm{L}}-E_{z}^{\mathrm{w}} \sin \psi\right]-\frac{\gamma v_{\perp}^{2}}{2 B_{0}} \frac{\mathrm{d} B_{0}}{\mathrm{~d} z}, \\
& \frac{\mathrm{d}\left(\gamma v_{\perp}\right)}{\mathrm{d} t}=\frac{e}{m_{0}}\left[\left(U_{\mathrm{R}}-v_{\|}\right) B_{\mathrm{R}}^{\mathrm{w}} \sin \zeta_{\mathrm{R}}+\left(U_{\mathrm{L}}-v_{\|}\right) B_{\mathrm{L}}^{\mathrm{w}} \sin \zeta_{\mathrm{L}}\right]+\frac{\gamma \nu_{\perp} v_{\|}}{2 B_{0}} \frac{\mathrm{d} B_{0}}{\mathrm{~d} z}, \\
& \frac{\mathrm{d} \phi}{\mathrm{d} t}=\frac{e}{\gamma m_{0}}\left[\frac{U_{\mathrm{R}}-v_{\|}}{v_{\perp}} B_{\mathrm{R}}^{\mathrm{w}} \cos \zeta_{\mathrm{R}}+\frac{U_{\mathrm{L}}-v_{\|}}{v_{\perp}} B_{\mathrm{L}}^{\mathrm{w}} \cos \zeta_{\mathrm{L}}-B_{z}^{\mathrm{w}} \cos \psi+B_{0}\right],
\end{aligned}
$$

where $\zeta_{\mathrm{R}}=\phi-\psi$ and $\zeta_{\mathrm{L}}=\phi+\psi$. When the wave amplitudes $B_{\mathrm{R}}^{\mathrm{W}}$ and $B_{\mathrm{L}}^{\mathrm{W}}$ are much smaller than the background magnetic field $B_{0},(140)$ is simplified as

$$
\frac{\mathrm{d} \phi}{\mathrm{d} t}=\frac{\Omega_{\mathrm{e}}}{\gamma} .
$$

The wave phase $\psi$ observed by an electron with a phase $\phi$ is given by

$$
\psi=\omega t-k_{z} z-k_{x} r_{\mathrm{c}} \sin \phi+\text { const. }=\psi_{\mathrm{B}}-\beta \sin \phi,
$$

where $\psi_{\mathrm{B}}$ is the wave phase at the center of the cyclotron motion given by $\psi_{\mathrm{B}}=\omega t-k_{z} z+$ const., $r_{\mathrm{c}}$ is the cyclotron radius given by $r_{\mathrm{c}}=\gamma \nu_{\perp} / \Omega_{\mathrm{e}}$, and $\beta=k_{x} r_{\mathrm{c}}$. Because of the perpendicular wave number $k_{x}$, the wave phase changes through the cyclotron motion around the guiding center. We expand the relative phase variation using the Bessel functions $J_{n}(\beta)$.

$$
\mathrm{e}^{i \zeta_{\mathrm{R}}}=\mathrm{e}^{i\left(\phi-\psi_{B}\right)} \mathrm{e}^{i \beta \sin \phi}=\mathrm{e}^{i\left(\phi-\psi_{B}\right)} \sum_{n=-\infty}^{\infty} J_{n}(\beta) \mathrm{e}^{i n \phi}=\sum_{n=-\infty}^{\infty} J_{n}(\beta) \mathrm{e}^{i(n+1) \phi-\psi_{B}}
$$

Shifting the index $n+1$ to $n$ in the summation and taking the imaginary parts, we have

$$
\sin \zeta_{\mathrm{R}}=\sum_{n=-\infty}^{\infty} J_{n-1}(\beta) \sin \left(n \phi-\psi_{B}\right) .
$$

Similarly we have
Substituting (144), (145), and (146) into (138) and (139), we obtain

$$
\begin{aligned}
& \frac{\mathrm{d}\left(\gamma v_{\|}\right)}{\mathrm{d} t}=\frac{1}{k_{z}} \sum_{n=-\infty}^{\infty} \omega_{t, n}^{2} \sin \zeta_{n}-\frac{\gamma v_{\perp}^{2}}{2 B_{0}} \frac{\mathrm{d} B_{0}}{\mathrm{~d} z}, \\
& \frac{\mathrm{d}\left(\gamma \nu_{\perp}\right)}{\mathrm{d} t}=\frac{1}{k_{z}} \sum_{n=-\infty}^{\infty} \omega_{s, n}^{2} \sin \zeta_{n}+\frac{\gamma \nu_{\perp} \nu_{\|}}{2 B_{0}} \frac{\mathrm{d} B_{0}}{\mathrm{~d} z},
\end{aligned}
$$

where

$$
\begin{aligned}
& \omega_{t, n}^{2}=\frac{e k_{z}}{m_{0}}\left[v_{\perp} B_{\mathrm{R}}^{\mathrm{w}} J_{n-1}(\beta)-v_{\perp} B_{\mathrm{L}}^{\mathrm{w}} J_{n+1}+E_{z}^{\mathrm{w}} J_{n}\right], \\
& \omega_{s, n}^{2}=\frac{e k_{z}}{m_{0}}\left[\left(U_{\mathrm{R}}-v_{\|}\right) B_{\mathrm{R}}^{\mathrm{w}} J_{n-1}-\left(U_{\mathrm{L}}-v_{\|}\right) B_{L}^{\mathrm{w}} J_{n+1}\right],
\end{aligned}
$$

and $\zeta_{n}$ is the generalized phase defined by $\zeta_{n}=n \phi-\psi_{B}$, which plays an important role in the nonlinear theory of the oblique whistler mode wave-particle interaction. Using the approximated equation (141), we take the time derivative of the generalized phase:

$$
\frac{\mathrm{d} \zeta_{n}}{\mathrm{~d} t}=n \frac{\mathrm{d} \phi}{\mathrm{d} t}-\frac{\mathrm{d} \psi_{B}}{\mathrm{~d} t}=\frac{n \Omega_{\mathrm{e}}}{\gamma}-\omega+k_{z} v_{\|} .
$$


The first-order resonance condition $\mathrm{d} \zeta_{n} / \mathrm{d} t=0$ gives the resonance velocity for the $n$-th order resonance:

$$
V_{\mathrm{R}, n}=\frac{\omega}{k_{z}}\left(1-\frac{n \Omega_{\mathrm{e}}}{\gamma \omega}\right) .
$$

We can rewrite (151) as

$$
\frac{\mathrm{d} \zeta_{n}}{\mathrm{~d} t}=k_{z}\left(v_{\|}-V_{\mathrm{R}, n}\right) .
$$

We calculate the second-order time derivative of $\zeta_{n}$ to analyze the nonlinear orbit of an electron nearly satisfying the first order resonance condition $v_{\|} \sim V_{\mathrm{R}, n}$.

$$
\frac{\mathrm{d}^{2} \zeta_{n}}{\mathrm{~d} t^{2}}=k_{z}\left(\frac{\mathrm{d} \nu_{\|}}{\mathrm{d} t}-\frac{\mathrm{d} V_{\mathrm{R}, n}}{\mathrm{~d} t}\right)
$$

where we have assumed the first order resonance condition $v_{\|}=V_{\mathrm{R}, n}$. To obtain the derivatives on the right hand side of (154), we calculate the variation of kinetic energy $K$ of an electron as

$$
\frac{\mathrm{d} \nu_{\perp}}{\mathrm{d} t}=\frac{1}{\gamma}\left(\frac{\omega_{s, n}^{2}}{k_{z}}-v_{\perp} \Omega_{d, n}\right) \sin \zeta_{n}+\frac{v_{\|} \nu_{\perp}}{2 \Omega_{\mathrm{e}}} \frac{\mathrm{d} \Omega_{\mathrm{e}}}{\mathrm{d} z} .
$$

We decompose the time variation of the $n$-th resonance velocity into several derivatives of variables in (152) as

$$
\begin{aligned}
\frac{\mathrm{d} V_{\mathrm{R}, n}}{\mathrm{~d} t}= & \frac{1}{k_{z}}\left(\frac{\mathrm{d} \omega}{\mathrm{d} t}-\frac{n v_{\|}}{\gamma} \frac{\partial \Omega_{\mathrm{e}}}{\partial z}+\frac{n \Omega_{\mathrm{e}}}{\gamma^{2}} \frac{\mathrm{d} \gamma}{\mathrm{d} t}\right) \\
& -\frac{1}{k_{z}^{2}}\left(\omega-\frac{n \Omega_{\mathrm{e}}}{\gamma}\right) \frac{\mathrm{d} k_{z}}{\mathrm{~d} t} .
\end{aligned}
$$

We assume quasi-parallel propagation $\sin ^{2} \Psi \ll 1$, where $\Psi$ is a wave normal angle given by $\Psi=\tan ^{-1}\left(k_{x} / k_{z}\right)$, under which the cold plasma dispersion relation remains basically the same as that of the parallel propagation. We have

$$
\frac{\mathrm{d} \omega}{\mathrm{d} t}=\left(1-\frac{v_{\|}}{V_{\mathrm{g} \|}}\right) \frac{\partial \omega}{\partial t} .
$$

$$
\begin{aligned}
\frac{\mathrm{d} K}{\mathrm{~d} t} & =-e \boldsymbol{E} \cdot \boldsymbol{v}=-e\left(E_{z}^{\mathrm{w}} v_{\|} \sin \psi-E_{\mathrm{R}}^{\mathrm{w}} v_{\perp} \sin \zeta_{\mathrm{R}}-E_{\mathrm{L}}^{\mathrm{w}} v_{\perp} \sin \zeta_{\mathrm{L}}\right) \\
& =e \sum_{n=-\infty}^{\infty}\left[v_{\|} E_{z}^{\mathrm{w}} J_{n}(\beta)+v_{\perp} E_{\mathrm{R}}^{\mathrm{w}} J_{n-1}(\beta)-v_{\perp} E_{\mathrm{L}}^{\mathrm{w}} J_{n+1}(\beta)\right] \sin \zeta_{n},
\end{aligned}
$$

where we expanded the phases by (144), (145), and (146). We obtain the time derivative of the Lorentz factor $\gamma$

$$
\frac{\mathrm{d} \gamma}{\mathrm{d} t}=\sum_{n=-\infty}^{\infty} \Omega_{d, n} \sin \zeta_{n}
$$

where

$$
\Omega_{d, n}=\frac{e}{c^{2} m_{0}}\left[\nu_{\|} E_{z}^{w} J_{n}(\beta)+v_{\perp} E_{\mathrm{R}}^{\mathrm{w}} J_{n-1}(\beta)-v_{\perp} E_{\mathrm{L}}^{\mathrm{w}} J_{n+1}(\beta)\right] .
$$

\subsection{Nonlinear dynamics at $\boldsymbol{n}$-th resonance}

We focus on the dynamics of an electron in the $n$-th resonance with the wave, i.e., $v_{\|} \sim V_{\mathrm{R}, n}$, and the wave amplitude is not extremely large so that the effects of the adjacent resonances $n \pm 1$ are negligible. Since most of the variations of the kinetic energy are due to the $n$-th order resonance, we have from (147) and (156)

$$
\frac{\mathrm{d} \nu_{\|}}{\mathrm{d} t}=\frac{1}{\gamma}\left(\frac{\omega_{t, n}^{2}}{k_{z}}-v_{\|} \Omega_{d, n}\right) \sin \zeta_{n}-\frac{v_{\perp}^{2}}{2 \Omega_{\mathrm{e}}} \frac{\mathrm{d} \Omega_{\mathrm{e}}}{\mathrm{d} z} .
$$

Similarly we obtain from (148)
From (32) and (10), we obtain

$$
\frac{\mathrm{d} k_{z}}{\mathrm{~d} t}=\frac{1}{V_{\mathrm{g} \|}}\left(1-\frac{v_{\|}}{V_{\mathrm{g} \|}}\right) \frac{\partial \omega}{\partial t}-\frac{\Lambda v_{\|} \chi \omega}{2 c \xi\left(\Omega_{\mathrm{e}}-\omega\right)} \frac{\partial \Omega_{\mathrm{e}}}{\partial z} .
$$

Substituting (156), (158), (160), (161), and (162) into (154), and setting $v_{\|}=V_{\mathrm{R}, n}$, we obtain the second-order derivative of the phase $\zeta_{n}$ as

$$
\frac{\mathrm{d}^{2} \zeta_{n}}{\mathrm{~d} t^{2}}=\Omega_{t, n}^{2}\left(\sin \zeta_{n}+S_{n}\right),
$$

where

$$
\begin{aligned}
\Omega_{t, n}^{2} & =\frac{1}{\gamma}\left(\omega_{t, n}^{2}-\omega \Omega_{d, n}\right), \\
S_{n}= & -\frac{1}{\Omega_{t, n}^{2}}\left\{\left(1-\frac{V_{\mathrm{R}, n}}{V_{\mathrm{g} \|}}\right)^{2} \frac{\partial \omega}{\partial t}\right. \\
& \left.+\left[\frac{\omega v_{\perp}^{2}}{2 \Omega_{\mathrm{e}} V_{\mathrm{P} \|}}-\frac{n}{\gamma} V_{\mathrm{R}, n}\left(1+\frac{\Lambda \chi^{2}\left[\Omega_{\mathrm{e}}-(\gamma / n) \omega\right]}{2\left(\Omega_{\mathrm{e}}-\omega\right)}\right)\right] \frac{\partial \Omega_{\mathrm{e}}}{\partial z}\right\} .
\end{aligned}
$$

Kinetic energy variation of energetic electrons trapped by the wave through the $n$-th resonance is given by the 

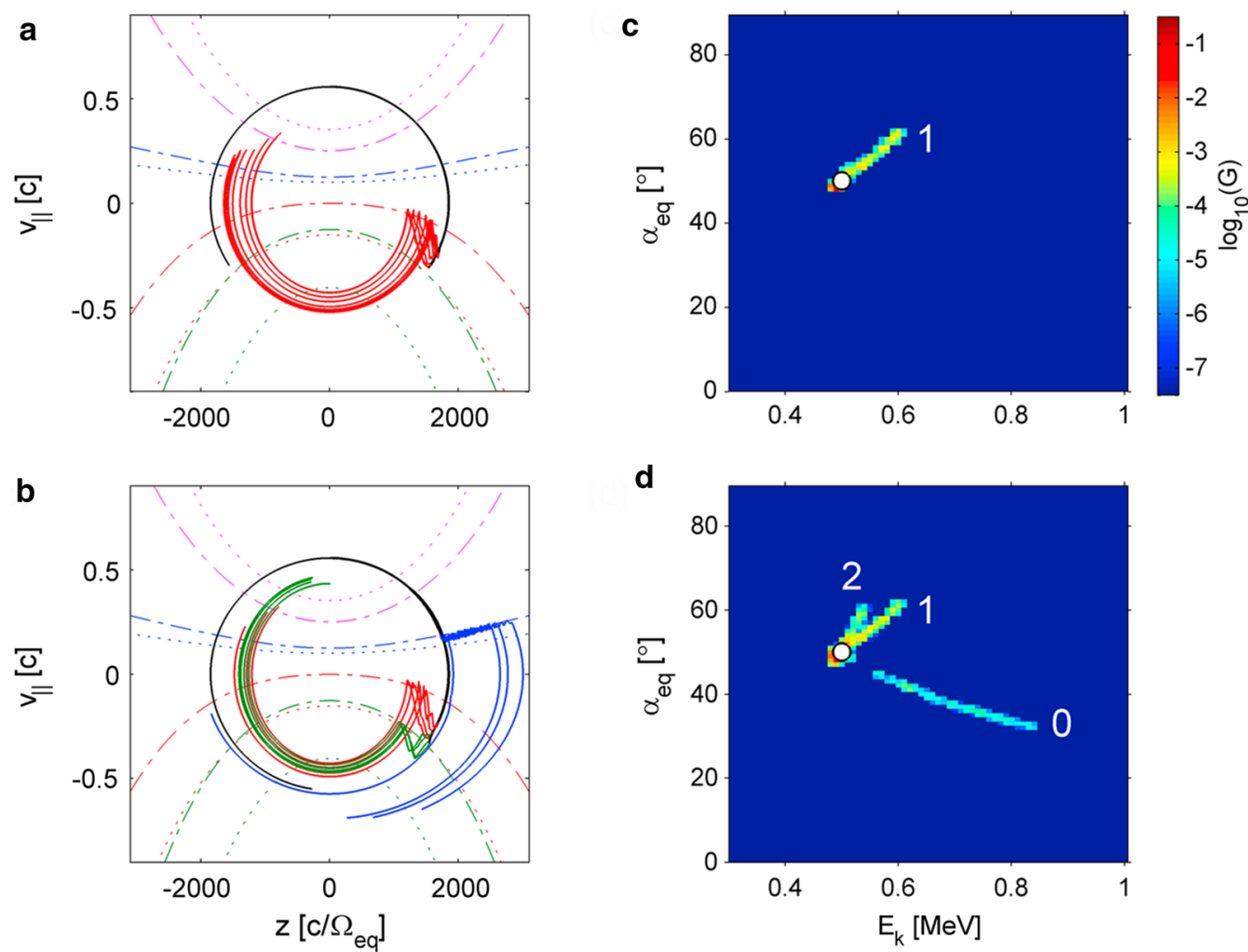

Fig. 14 Trajectories of trapped resonant electrons. Acceleration of electrons through nonlinear trapping by a chorus wave with the wave normal angle parallel $(\mathbf{a}, \mathbf{c})$ and oblique $(\mathbf{b}, \mathbf{d})$ to the background magnetic field. In $\mathbf{a}, \mathbf{b}$, resonance velocities for $n=0,1,2$ resonances are plotted in dash-dot lines $\left(\omega=0.5 \Omega_{\mathrm{e}}\right)$ and dotted lines $\left(\omega=0.2 \Omega_{\mathrm{e}}\right.$ ). Going through the resonances, colors of the trajectories changes from black to blue, red, and green, corresponding to $n=0,1,2$ resonances. The numbers next to the branches of the Green's functions represent the $n$-th resonances (after Hsieh and Omura (2018))

following equation obtained from (155) and (165) under the second-order resonance condition $\sin \zeta_{n}+S_{n}=0$.

$$
\frac{\mathrm{d} K_{n}}{\mathrm{~d} t}=-m_{0} c^{2} \Omega_{d, n} S_{n}
$$

For all possible resonances with the oblique whistler mode wave $(n=-\infty \sim \infty)$, this equation is valid as far as $\left|S_{n}\right| \leq 1$, while most of the acceleration is due to resonances with $n=-1 \sim 2$ as presented in test particle simulations by Hsieh and Omura (2017). This is also because the term in $S_{n}$ containing $n / \gamma$ becomes large for a large number $n$ except for relativistic electrons with a large $\gamma$. The energy variation of resonant electrons trapped by the wave potential is due to the frequency variation $\partial \omega / \partial t$ and the gradient of the magnetic field. Comparing simulation runs between purely parallel propagation and gradual oblique propagation, there occur significant differences in the trajectories of energetic electrons especially with energies greater than several hundred $\mathrm{keV}$. As shown by numerical Green's functions (Omura et al. 2015b) in Fig. 14, we find that the acceleration efficiency of Landau resonant $(n=0)$ electrons is very high in comparison with the cyclotron resonant $(n=1)$ electrons. However, most of the acceleration is due to the perpendicular electric field rather than the parallel electric field even in the Landau resonance (Hsieh and Omura 2017; Omura et al. 2019). The Green's function is calculated by tracing dynamics of 28,800 electrons in a chorus wave model with frequency variation from $0.2 \Omega_{\mathrm{e}}$ to $0.5 \Omega_{\mathrm{e}}$. In the oblique propagation the wave normal angle gradually changes from 0 to 60 degrees as the chorus wave packet propagates from the equator to higher latitudes.

\subsection{Nonlinear damping at half the cyclotron frequency}

In the case of quasi-parallel propagation, the group velocity and the phase velocity become equal near half the cyclotron frequency, in which the Landau resonance becomes significant because both waves and resonant electrons see stationary phases and the wave amplitude. Using (112) and (117), we calculate $\Delta V=\left|V_{\mathrm{p} \|}-V_{\mathrm{g} \|}\right|$ as a function of frequency $\omega$ and wave normal angle $\theta_{k B}$. When $\Delta V \sim 0$, the wave phase in the wave packet 
becomes stationary, and electrons moving with velocities close to the parallel phase velocity $V_{\mathrm{p} \|}$ can be trapped by the wave potential. Therefore, a very effective wave-particle interaction can take place for the Landau resonance near half the cyclotron frequency. We assume a chorus wave packet generated near the equator and propagating to higher latitudes. The wave normal angle of chorus waves is nearly zero near the equator, and it gradually becomes oblique through propagation because of the curvature of the background magnetic field. Hsieh and Omura (2018) has conducted test particle simulations to find formation of resonant current through Landau resonance, and found $\boldsymbol{J}_{\mathrm{R}} \cdot \boldsymbol{E}_{\mathrm{w}}>0$. The resonant current is due to nonlinear trapping of Landau resonant electrons in a wave potential formed by the parallel electric field component $E_{\mathrm{w} \|}$ and $\boldsymbol{v}_{\perp} \times \boldsymbol{B}_{\mathrm{R}}$. Because of the frequency sweep rate $\partial \omega / \partial t>0$ and the gradient of the background magnetic field $\partial \Omega_{\mathrm{e}} / \partial z>0$ in (165), we have a negative inhomogeneity factor $S_{n}<0$, which distorts the wave potential shifting the stable equilibrium point to a phase giving $-e \boldsymbol{E}_{\mathrm{R}} \cdot \boldsymbol{v}_{\perp}>0$ for a stably trapped resonant electron. The trapped electrons are accelerated effectively especially at half the local cyclotron frequency where the phase velocity and the group velocity are nearly equal for the quasi-parallel propagation. The wave packet with a frequency close to half the cyclotron frequency gives energy to the resonant electrons, undergoing the decrease of the wave amplitude. Since the damping takes place locally at half the cyclotron frequency along the magnetic field line, the frequency of the most effective damping increases as the wave packet moves away from the magnetic equator. The damping along the magnetic field line results in formation of a gap between the lower-band and upper-band chorus emissions. The formation of the gap is schematically illustrated in Fig. 2 of Yagitani et al. (2014).

As another possible mechanism to generate upperband chorus emissions, a nonlinear wave process called the lower band cascade has been studied by observations, simulation, and theory (Gao et al. 2018). When the wave amplitudes of the lower-band chorus emissions become very large, the proposed nonlinear process may take place, while the nonlinear damping process at half the cyclotron frequency takes place as far as a coherent wave propagates obliquely with a small wave normal angle.

\section{Summary and discussion}

The following topics have been clarified along with the derivation of the formulas that have not been published previously.
- The condition for resonant electrons to be free from the anomalous trapping at lower pitch angles is given by (26).

- The adiabatic variation of a subtracted-Maxwellian momentum distribution function is described by (54)-(60), which can be applied for the calculation of the nonlinear growth rate at a position away from the equator.

- The inhomogeneity factor $S_{n}$ given by (165) for the $n$-th resonance in the oblique propagation is derived under the assumption of quasi-parallel propagation. The formula of $S_{n}$ contains the frequency sweep rate as observed at a fixed point and the gradient of the magnetic field. Along with the second-order resonance condition, we have obtained the formula for acceleration of trapped resonant electrons through the $n$-th resonance.

- The nonlinear growth rates, optimum wave amplitude, threshold wave amplitude, and chorus equations in the previous publications contained inconsistent powers of the Lorentz factor $\gamma$, and these formulas are corrected.

The theoretical developments presented in this paper are based on our analyses of simulations reproducing chorus and hiss emissions, and they are mostly confirmed by the observations. In the simulations Maxwell's equations are solved along with the calculation of particle dynamics under the self-consistent electromagnetic fields. Principles of these electromagnetic processes are simple, and the nonlinear dynamics of resonant particles in a coherent wave potential is well understood. However, the combination of the nonlinear trajectories of energetic particles and the generation process of new wave fields with varying frequency and wave numbers involves rapid variation of the wave phase and amplitudes, and they are still difficult to be understood completely. In this respect, some of the equations that we obtained, such as the chorus equations, are still phenomenological, and they do not describe the detailed physical processes of the wave generation.

The gradient of the background magnetic field controls the threshold wave amplitude, and the size of the nonlinear interaction region around the magnetic equator. A very efficient convective wave growth occurs with a balance of the growing wave amplitude and the increasing gradient of the magnetic field. The mechanism works for electron holes with depletion of trapped resonant electrons in the velocity phase space, which originally is formed at the moment of wave packet generation with rising-tone frequency. Therefore, we have more observations of rising tone emissions in the magnetosphere. We occasionally observe falling-tone emissions which are 
generated by an electron hill formed by trapped electrons in the velocity phase as demonstrated by simulations (Nunn and Omura 2012; Nogi et al. 2020). However, we have not obtained the threshold wave amplitude for the generation of falling-tone emissions.

One of the elements that makes the analyses difficult is the electromagnetic fluctuations in the simulation as well as in the real plasma environment in the magnetosphere. Since the nonlinear wave growth process is induced by a finite amplitude wave above the threshold level, the wave growth from the thermal fluctuations to the threshold amplitude should be evaluated properly. However the level of electromagnetic fluctuations is also contributed by energetic electrons being injected. Studies on the thermal fluctuations in simulations and in the real plasma environment are necessary. In the magnetosphere, external waves such as whistlers and those from VLF transmitters on the ground, or waves from other parts of the magnetosphere may work as triggering waves for the nonlinear process. Active wave transmission from spacecraft near the magnetic equator would be very ineresting and useful for better understanding of the nonlinear triggering process of chorus emissions.

Chorus emissions are generated by electrons with energy of $10-100 \mathrm{keV}$ in the Earth's magnetosphere, precipitating them into the polar atmosphere, while electrons at higher energy ranges are accelerated efficiently to $\mathrm{MeV}$ energy contributing to rapid formation of the outer radiation belt (Omura et al. 2015b; Kubota and Omura 2018; Hsieh et al. 2020). These interesting physical processes of relativistic electron acceleration are not included in the present paper. Rapid variations of the radiation belts are also due to occurrence of EMIC emissions with risingtone frequency variations (Omura and Zhao 2012, 2013). The mechanism of the EMIC emissions is nearly the same as chorus emissions (Omura et al. 2010; Shoji and Omura 2013). A summary report on nonlinear processes of relativistic electrons and EMIC waves is left as a future work.

\footnotetext{
Acknowledgements

The theory and simulations presented in this manuscript are based on fruitful discussions with David Nunn, Danny Summers, Yuto Katoh, Mitsuru Hikishima, Satoshi Yagitani, Craig Kletzing, Masafumi Shoji, Satoko Nakamura, Yi-Kai Hsieh Yuko Kubota, Takeshi Nogi, Miroslav Hanzelka, Miwa Tobita, Yin Liu, Li Li, John Foster, and Philip Erickson. This work was supported by JSPS KAKENHI Grant $17 \mathrm{H} 06140$.
}

\section{Author's contributions}

YO has contributed to the entire content of this paper. The author read and approved the final manuscript.

\section{Author's information}

YO has been working on theory and simulations of nonlinear wave-particle interaction for 40 years at Kyoto University. YO received Zeldovich Award of COSPAR (1991), Tanakadate Award of SGEPSS (1996), Science and Technology Award, MEXT Japan (2006), and Appleton Prize of URSI (2017). YO was the president (2019-2021) of the Society of Geomagnetism and Earth, Planetary and Space Sciences (SGEPSS).
Received: 24 November 2020 Accepted: 12 February 2021

Published online: 20 April 2021

\section{References}

Bell TF (1984) The nonlinear gyroresonance interaction between energetic electrons and coherent VLF waves propagating at an arbitrary angle with respect to the Earth's magnetic field. J Geophys Res 89(A2):905-918. https://doi.org/10.1029/JA089iA02p00905

Bortnik J, Thorne RM, Meredith NP (2008) The unexpected origin of plasmaspheric hiss from discrete chorus emissions. Nature 452(7183):62-66. https://doi.org/10.1038/nature06741

Gao X, Lu Q, Wang S, Wang S (2018) Theoretical analysis on lower band cascade as a mechanism for multiband chorus. AIP Adv 8:055003. https:// doi.org/10.1063/1.5025507

Golkowski M, Harid V, Hosseini P (2019) Review of controlled excitation of non-linear wave-particle interactions in the magnetosphere. Front Astron Space Sci 6:2. https://doi.org/10.3389/fspas.2019.00002

Foster JC, Erickson PJ, Omura Y, Baker DN, Kletzing CA, Claudepierre SG (2017) Van Allen Probes observations of prompt MeV radiation belt electron acceleration in nonlinear interactions with VLF chorus. J Geophys Res Space Phys 122:324-339. https://doi.org/10.1002/2016JA023429

Hanzelka M, Santolik O, Omura Y, Kolmasova I, Kletzing CA (2020) A model of the subpacket structure of rising tone chorus emissions. J Geophys Res Space Phys 125:e2020JA028094. https://doi.org/10.1029/2020JA028094

Helliwell RA (1993) Whistlers and related ionospheric phenomena. Dover Publicaitons, New York

Hikishima M, Yagitani S, Omura Y, Nagano I (2009) Full particle simulation of whistler-mode rising chorus emissions in the magnetosphere. J Geophys Res 114:A01203. https://doi.org/10.1029/2008JA013625

Hikishima M, Omura Y, Summers D (2010) Self-consistent particle simulation of whistler mode triggered emissions. J Geophys Res 115:A12246. https:// doi.org/10.1029/2010JA015860

Hikishima M, Omura Y (2012) Particle simulations of whistler-mode rising-tone emissions triggered by waves with different amplitudes. J Geophys Res 117:A04226. https://doi.org/10.1029/2011JA017428

Hikishima M, Omura Y, Summers D (2020) Particle simulation of the generation of plasmaspheric hiss. J Geophys Res Space Phys 125:e2020JA027973. https://doi.org/10.1029/2020JA027973

Hiraga R, Omura Y (2020) Acceleration mechanism of radiation belt electrons through interaction with multisubpacket chorus waves. Earth Planets Space 72:21. https://doi.org/10.1186/s40623-020-1134-3

Hsieh Y-K, Omura Y (2017) Nonlinear dynamics of electrons interacting with oblique whistler mode chorus in the magnetosphere. J Geophys Res Space Phys 122:675-694. https://doi.org/10.1002/2016JA023255

Hsieh Y-K, Omura Y (2018) Nonlinear damping of oblique whistler mode waves via Landau resonance. J Geophys Res Space Phys 123:7462-7472. https:// doi.org/10.1029/2018JA025848

Hsieh Y-K, Kubota Y, Omura Y (2020) Nonlinear evolution of radiation belt electron fluxes interacting with oblique whistler mode chorus emissions. J Geophys Res Space Phys 125:e2019JA027465. https://doi.org/10.1029/ 2019JA027465

Katoh Y, Omura Y (2006) A study of generation mechanism of VLF triggered emission by self-consistent particle code. J Geophys Res 111:A12207. https://doi.org/10.1029/2006JA011704

Katoh Y, Omura Y (2007) Computer simulation of chorus wave generation in the Earth's inner magnetosphere. Geophys Res Lett 34:L03102. https:// doi.org/10.1029/2006GL028594

Katoh Y, Omura Y (2011) Amplitude dependence of frequency sweep rates of whistler mode chorus emissions. J Geophys Res 116:A07201. https://doi. org/10.1029/2011JA016496

Katoh Y, Omura Y (2013) Effect of the background magnetic field inhomogeneity on generation processes of whistler-mode chorus and broadband hiss-like emissions. J Geophys Res 118:4189-198. https://doi.org/10.1002/ jgra.50395

Katoh Y, Omura Y (2016) Electron hybrid code simulation of whistler-mode chorus generation with real parameters in the Earth's inner magnetosphere. Earth Planets Space 68:192. https://doi.org/10.1186/ s40623-016-0568-0 
Katoh Y, Omura Y, Miyake Y, Usui H, Nakashima H (2018) Dependence of generation of whistler mode chorus emissions on the temperature anisotropy and density of energetic electrons in the Earth's inner magnetosphere. J Geophys Res Space Phys. https://doi.org/10.1002/2017JA024801

Ke Y, Gao X, Lu Q, Wang X, Wang S (2017) Generation of rising-tone chorus in a two-dimensional mirror field by using the general curvilinear PIC code. J Geophys Res Space Phys. https://doi.org/10.1002/2017JA024178

Kitahara M, Katoh Y (2019) Anomalous trapping of low pitch angle electrons by coherent whistler mode waves. J Geophys Res Space Phys 124:55685583. https://doi.org/10.1029/2019JA026493

Kubota Y, Omura Y (2017) Rapid precipitation of radiation belt electrons induced by EMIC rising tone emissions localized in longitude inside and outside the plasmapause. J Geophys Res Space Phys 122:293-309. https://doi.org/10.1002/2016JA023267

Kubota Y, Omura Y (2018) Nonlinear dynamics of radiation belt electrons interacting with chorus emissions localized in longitude. J Geophys Res Space Phys 123:4835-4857. https://doi.org/10.1029/2017JA025050

Kurita S, Katoh Y, Omura Y, Angelopoulos V, Cully CM, Le Contel O, Misawa H (2012) THEMIS observation of chorus elements without a gap at half the gyrofrequency. J Geophys Res 117:A11223. https://doi.org/10.1029/2012J A018076

Lu Q, Ke Y, Wang X, Liu K, Gao X, Chen L, Wang S (2019) Two dimensional general curvilinear particle in cell (gCPIC) simulation of rising tone chorus waves in a dipole magnetic field. J Geophys Res Space Phys. https://doi. org/10.1029/2019JA026586

Nakamura S, Omura Y, Summers D, Kletzing CA (2016) Observational evidence of the nonlinear wave growth theory of plasmaspheric hiss. Geophys Res Lett 43:10040-10049. https://doi.org/10.1002/2016GL070333

Nakamura S, Omura Y, Summers D (2018) Fine structure of whistler mode hiss in plasmaspheric plumes observed by the Van Allen Probes. J Geophys Res Space Phys 123:9055-9064. https://doi.org/10.1029/2018JA025803

Nogi T, Nakamura S, Omura Y (2020) Full particle simulation of whistler-mode triggered falling-tone emissions in the magnetosphere. J Geophys Res Space Phys 125:e2020JA027953. https://doi.org/10.1029/2020JA027953

Nunn D, Omura Y (2012) A computational and theoretical analysis of falling frequency VLF emissions. J Geophys Res 117:A08228. https://doi.org/10. 1029/2012JA017557

Nunn D, Omura Y (2015) A computational and theoretical investigation of nonlinear wave-particle interactions in oblique whistlers. J Geophys Res Space Phys 120:2890-2911. https://doi.org/10.1002/2014JA020898

Omura Y, Nunn D, Matsumoto H, Rycroft MJ (1991) A review of observational, theoretical and numerical studies of VLF triggered emissions. J Atmos Terr Phys 53:351-368

Omura Y, Katoh Y, Summers D (2008) Theory and simulation of the generation of whistler-mode chorus. J Geophys Res 113:A04223. https://doi.org/10. 1029/2007JA012622

Omura Y, Hikishima M, Katoh Y, Summers D, Yagitani S (2009) Nonlinear mechanisms of lower band and upper band VLF chorus emissions in the magnetosphere. J Geophys Res 114:A07217. https://doi.org/10.1029/2009JA014206

Omura Y, Pickett J, Grison B, Santolik O, Dandouras I, Engebretson M, Dcrau PME, Masson A (2010) Theory and observation of electromagnetic ion cyclotron triggered emissions in the magnetosphere. J Geophys Res 115:A07234. https://doi.org/10.1029/2010JA015300

Omura Y, Nunn D (2011) Triggering process of whistler mode chorus emissions in the magnetosphere. J Geophys Res 116:A05205. https://doi.org/10. 1029/2010JA016280
Omura Y, Zhao Q (2012) Nonlinear pitch angle scattering of relativistic electrons by EMIC waves in the inner magnetosphere. J Geophys Res 117:A08227. https://doi.org/10.1029/2012JA017943

Omura Y, Zhao Q (2013) Relativistic electron microbursts due to nonlinear pitch angle scattering by EMIC triggered emissions. J Geophys Res Space Phys 118:5008-5020. https://doi.org/10.1002/jgra.50477

Omura Y, Nakamura S, Kletzing CA, Summers D, Hikishima M (2015a) Nonlinear wave growth theory of coherent hiss emissions in the plasmasphere. J Geophys Res Space Phys 120:7642-7657. https://doi.org/10.1002/2015J A021520

Omura Y, Miyashita Y, Yoshikawa M, Summers D, Hikishima M, Ebihara Y, Kubota Y (2015b) Formation process of relativistic electron flux through interaction with chorus emissions in the Earth's inner magnetosphere. J Geophys Res Space Phys 120:9545-9562. https://doi.org/10.1002/2015J A021563

Omura Y, Hsieh Y-K, Foster JC, Erickson PJ, Kletzing CA, Baker DN (2019) Cyclotron acceleration of relativistic electrons through Landau resonance with obliquely propagating whistler-mode chorus emissions. J Geophys Res Space Phys 124:2795-2810. https://doi.org/10.1029/2018JA026374

Santolik O, Kletzing CA, Kurth WS, Hospodarsky GB, Bounds SR (2014) Fine structure of large-amplitude chorus wave packets. Geophys Res Lett. https://doi.org/10.1002/2013GL058889

Shoji M, Omura Y (2013) Triggering process of electromagnetic ion cyclotron rising tone emissions in the inner magnetosphere. J Geophys Res Space Phys 118:5553-5561. https://doi.org/10.1002/jgra.50523

Stix TH (1992) Waves in plasmas. American Institute of Physics, College Park

Tao X (2014) A numerical study of chorus generation and the related variation of wave intensity using the DAWN code. J Geophys Res Space Phys. https://doi.org/10.1002/2014JA019820

Tao X, Zonca F, Chen L, Wu Y (2020) Theoretical and numerical studies of chorus waves: a review. Sci China Earth Sci 63:78-92. https://doi.org/10. 1007/s11430-019-9384-6

Sugiyama H, Singh S, Omura Y, Shoji M, Nunn D, Summers D (2015) Electromagnetic ion cyclotron waves in the Earth's magnetosphere with a kappa-Maxwellian particle distribution. J Geophys Res Space Phys 120:8426-8439. https://doi.org/10.1002/2015JA021346

Summers D, Omura Y, Miyashita Y, Lee D-H (2012) Nonlinear spatiotemporal evolution of whistler mode chorus waves in Earth's inner magnetosphere. J Geophys Res 117:A09206. https://doi.org/10.1029/2012JA017842

Summers D, Omura Y, Nakamura S, Kletzing CA (2014) Fine structure of plasmaspheric hiss. J Geophys Res Space Phys. https://doi.org/10.1002/ 2014JA020437

Yagitani S, Habagishi T, Omura Y (2014) Geotail observation of upper band and lower band chorus elements in the outer magnetosphere. J Geophys Res Space Phys. https://doi.org/10.1002/2013JA019678

Yoon YD, Bellan PM (2020) Nondiffusive pitch-angle scattering of a distribution of energetic particles by coherent whistler waves. J Geophys Res Space Phys 125:e2020JA027796. https://doi.org/10.1029/2020JA027796

\section{Publisher's Note}

Springer Nature remains neutral with regard to jurisdictional claims in published maps and institutional affiliations. 